TRANSACTIONS OF THE

AMERICAN MATHEMATICAL SOCIETY

Volume 350, Number 5, May 1998, Pages 2009-2031

S 0002-9947(98)01929-1

\title{
COMPOSITION FACTORS OF INDECOMPOSABLE MODULES
}

\author{
MARIA IZABEL RAMALHO MARTINS
}

\begin{abstract}
Let $\Lambda$ be a connected, basic finite dimensional algebra over an algebraically closed field. Our main aim is to prove that if $\Lambda$ is biserial, its ordinary quiver has no loop and every indecomposable $\Lambda$-module is uniquely determined by its composition factors, then each indecomposable $\Lambda$-module is multiplicity-free.
\end{abstract}

In this article a $k$-algebra $\Lambda$ means a finite dimensional $k$-algebra, where $k$ is a fixed algebraically closed field. Denote by $\Lambda$-mod the category of all finitely generated left $\Lambda$-modules and by $\Lambda$-ind the full subcategory of $\Lambda$-mod defined by one representative of each isomorphism class of indecomposable $\Lambda$-modules. All modules and maps are in $\Lambda$-mod. A module $M$ is called multiplicity-free if its composition factors are pairwise non-isomorphic.

We say that $\Lambda$-ind is determined by composition factors (multiplicity-free) if every module in $\Lambda$-ind is uniquely determined by its composition factors (respectively, multiplicity-free). For example, it is well-known that an algebra $\Lambda$ is semisimple if and only if each of its modules is uniquely determined by composition factors. Though the composition factors do not determine arbitrary modules over nonsemisimple algebras, it is an interesting question to know under which conditions it has the property that $\Lambda$-ind is determined by composition factors. In [5], Auslander and Reiten obtained sufficient conditions for $\Lambda$-ind to be determined by composition factors. Besides this question it is also an interesting question to know when an algebra has the property that $\Lambda$-ind is multiplicity free, and so find out if there is any relationship between those properties. It is well-known, for example, that if $\Lambda$ is a representation-finite hereditary $k$-algebra then $\Lambda$-ind is determined by composition factors (see [2]), although it may not hold true in general that " $\Lambda$-ind is multiplicity-free". It is not difficult to show that, among the representation-finite hereditary $k$-algebras, the ones of the form $\Lambda=k Q$, with $\Lambda$-ind multiplicity-free, coincide with those whose quiver $Q$ is of type $A_{n}$.

According to [12], if $\Lambda$ is a $k$-algebra such that $\Lambda$-ind is multiplicity-free, then $\Lambda$ is a representation-finite biserial and Schurian algebra. Recall that an algebra $\Lambda$ is biserial if the radical of any indecomposable non-uniserial projective, left or right, $\Lambda$-module is a sum of two uniserial submodules whose intersection is simple or zero. Well-known examples of biserial algebras are Nakayama algebras and iterated tilted algebras of type $A_{n}$ (see [1] for the definition).

With respect to a relationship between the properties $\Lambda$-ind being determined by composition factors and $\Lambda$-ind being multiplicity-free, the results of Pogorzaly and Skowroński (see [12], th.1) together with results of Skowroński and Waschbüsch (see

Received by the editors September 19, 1995 and, in revised form, August 1, 1996.

1991 Mathematics Subject Classification. Primary 16G20, 16 G60.

(C)1998 American Mathematical Society 
[14], cor. of th.1) show that the conditions (i) $\Lambda$ is biserial and $\Lambda$-ind is determined by composition factors and (ii) $\Lambda$-ind is multiplicity-free are equivalent, if $\Lambda$ is assumed to be a factor of a hereditary algebra. (Recall that a $k$-algebra $\Lambda$ is a factor of a hereditary algebra if and only if the corresponding ordinary quiver has neither loops nor oriented cycles.)

This leads us to the following questions: for which $k$-algebras does the implication (i) $\Rightarrow$ (ii) or (ii) $\Rightarrow$ (i) hold? Can these hold even if $\Lambda$ is not a factor of a hereditary algebra? With respect to the implication (ii) implies (i) the answer is not always. We shall exhibit, in section 3 , an example where $\Lambda$ is a biserial $k$-algebra, not Nakayama, such that (ii) is satisfied but (i) is not. (It is very simple to find a Nakayama algebra with such properties).

The answer to the implication (i) implies (ii) is the main aim of this article, and it is given by the following theorem.

Theorem 6.2. Let $\Lambda$ be a connected, basic finite dimensional algebra over an algebraically closed field, whose ordinary quiver has no loop. Assume that $\Lambda$-ind is determined by composition factors. Then $\Lambda$-ind is multiplicity-free.

In order to formulate the main result we used the diagramatic structure of the representation-finite biserial $k$-algebras $\Lambda$ given by Skowroński and Pogorzaly (in $[12]$, th.2). We carry this structure over to a certain "local" structure of some indecomposable modules, in the case that the ordinary quiver $Q$ of $\Lambda$ has no loop and all oriented cycles in $k Q$ are in the relation set; and this allows us to prove an interesting result which is useful for the proof of theorem 6.2 , which is the following theorem.

Theorem 6.1. Let $\Lambda=k\left(Q_{\Lambda}, R_{\Lambda}\right)$ be a representation-finite biserial bound quiver algebra. Suppose that the quiver $Q_{\Lambda}$ has no loop and that all oriented cycles of $k Q_{\Lambda}$ are in $R_{\Lambda}$. If $M$ is a multiplicity-free indecomposable $\Lambda$-module, then either $M$ is projective-injective non-uniserial or $M$ is of type $A_{n}$.

As a consequence of theorems 6.1 and 6.2 we have that if $\Lambda$ is a biserial $k$ algebra whose ordinary quiver has no loop, and such that $\Lambda$-ind is determined by composition factors, then there are two types of indecomposable modules: the projective-injective non-uniserial modules and the modules of type $A_{n}$.

The results in this article are part of my Ph.D. thesis, written under Professor Hector A. Merklen at University of São Paulo. I thank Professor Merklen for very stimulating discussions and for his useful remarks during my work.

\section{Preliminaries}

Given a $k$-algebra $\Lambda$ and a module $M$, we denote by $K_{0}(\Lambda)$ the Grothendieck group of $\Lambda$ and by $[M]$ the image of $M$ in $K_{0}(\Lambda)$. We will identify a $k$-algebra $\Lambda$ with its associated bound quiver algebra $k\left(Q_{\Lambda}, R_{\Lambda}\right)$ (see [8], [9]), where $Q_{\Lambda}$ denotes the ordinary quiver of $\Lambda$. Consequently we will identify the category $\Lambda$ mod with the category $\left(Q_{\Lambda}, R_{\Lambda}\right)$-mod of the finite $k$-representations of $\left(Q_{\Lambda}, R_{\Lambda}\right)$ ([8], [9]). This enables us to denote by $P_{a}\left(I_{a}\right)$ the indecomposable projective (injective) module which corresponds to the vertex $a$ of $Q_{\Lambda}$, and by $S_{a}$ the simple module associated to the vertex $a$. If $(Q, R)$ is a quiver with relations, we denote by $Q^{o p}$ the quiver obtained from $Q$ by reversing arrows and by $\gamma^{*}$ the arrows of $Q^{o p}$ to which correspond the arrows $\gamma$ of $Q$. So $\left(Q^{o p}, R^{o p}\right)$ is the opposite quiver with relations of $(Q, R)$, where $R^{o p}=\left\{\rho^{*} \in k Q^{o p}: \rho \in R\right\}$. To each module $M$ 
we associate a subquiver of $Q_{\Lambda}$, which is denoted by $Q_{M}$, as follows. The set of vertices of $Q_{M}$ is the set of the vertices $x$ of $Q_{\Lambda}$ for which $M(x) \neq(0)$, and its set of arrows is formed by the arrows $\alpha$ of $Q_{\Lambda}$ for which $M(\alpha) \neq 0$. Sometimes we refer to the set of vertices of the quiver of $M$ as the support of $M$ and denote it by supp $M$. It is easy to verify that if $M$ is indecomposable, then $Q_{M}$ is connected. We say that a module $M$ is of type $A_{n}$ if the underlying graph of the quiver $Q_{M}$ is a diagram $A_{n}$ and if $M(x) \cong k$, for each $x \in \operatorname{supp} M$, and the $k$-morphism $M(\alpha)$ is a scalar multiple of the identity, for each arrow $\alpha$ of $Q_{M}$. For an algebra $\Lambda$ and any $\Lambda$-module $M$ we will denote by $I_{M}$ the $\Lambda$-injective envelope of $M$, by top $M$ the top of $M$, by socM the socle of $M$, by $\operatorname{rad} M$ the radical of $M$, and by $l(M)$ the length of $M$. Finally, by $D$ we denote the duality $\operatorname{Hom}_{k}(-, k)$, by $\tau$ we denote the Auslander-Reiten operator which maps the non-projective indecomposables one-toone to the non-injective indecomposables (it has a natural inverse $\tau^{-1}$ ), and by $\Gamma_{\Lambda}$ the Auslander-Reiten quiver of $\Lambda$ (see [3], [4], or [9]).

Remark 1.1. Let $\Lambda$ be a $k$-algebra such that $\Lambda$-ind is determined by composition factors. Then $\Lambda$ is representation-finite. This is an immediate consequence of BT II (see [7], [11] or [13]). It is a nice problem to prove this without using BT II. It follows from this that the ordinary quiver of $\Lambda$ has neither multiple arrows nor diagrams $\tilde{A_{n}}$ without relations, where $n \geq 3$.

\section{Nakayama ALGebras}

In this section we consider our questions in the easy case of Nakayama algebras. Recall that for a Nakayama algebra $\Lambda$, by definition, all indecomposable projective and injective $\Lambda$-modules are uniserial. In particular, in this section the algebras mentioned are Artin algebras (that is, an artinian ring $\Lambda$ with 1 such that its centre contains an artinian subring over which $\Lambda$ is finitely generated as a module). We refer to [2], [6] and [8] for well known facts that we shall use here freely. Of course, as $\Lambda$ is assumed connected, we assume that all simple $\Lambda$-modules lie in one $\tau$-orbit. Thus, we can also order the representatives $P_{1}, P_{2}, \ldots, P_{n}$ of all indecomposable projective modules according to the Kupisch series (see [6]), that is, in such a way that top $P_{i+1} \cong \tau^{-1}\left(\right.$ top $\left.P_{i}\right)$ for $i=1,2, \ldots, n-1$ and, if $l\left(P_{1}\right) \neq 1$, top $P_{1} \cong$ $\tau^{-1}\left(\right.$ top $\left.P_{n}\right)$.

Proposition 2.1. Let $\Lambda$ be a connected, basic Nakayama algebra and $n$ be the rank of the Grothendieck group of $\Lambda$. If $n=1$ or $n \geq 2$ and there is a simple projective $\Lambda$-module, then $\Lambda$-ind is always determined by composition factors. If, on the contrary, $n \geq 2$ and $l(P) \geq 2$ for all indecomposable projective $\Lambda$-module, then $\Lambda$-ind is determined by composition factors if and only if $l(P) \leq n$, for all indecomposable projective $P$, and there is at most one of them of length $n$.

Proof. The first of these assertions is an easy consequence of well known properties related to the Kupisch series of $\Lambda$, and the same is the case for the "only if" part of the last assertion.

Let us assume now that $l(M) \leq n$, for all $M$ in $\Lambda$-ind. If $M$ is not projective, then $l(M)<n$ and, since it is determined by its top and length, $M$ is uniquely determined by its composition factors. But this is clearly true for any indecomposable projective $\Lambda$-module $P$ with $l(P)<n$. Hence the result follows.

When $\Lambda$ is a Nakayama algebra, we also establish necessary and sufficient conditions for $\Lambda$-ind to be multiplicity-free. 
Proposition 2.2. Let $\Lambda$ be a connected, basic Nakayama algebra and $n$ be the rank of the Grothendieck group of $\Lambda$. Then $\Lambda$-ind is multiplicity-free if and only if every projective indecomposable $\Lambda$-module has length at most $n$.

Proof. This is an immediate consequence of properties related to the Kupisch series and the fact that every indecomposable $\Lambda$-module is local.

\section{Some CONDitions For $\Lambda$-IND to Be DETERMined By COMPOSition FACTORS}

In this section we will establish some necessary conditions for $\Lambda$-ind to be determined by composition factors, in the case that $\Lambda$ is a bound quiver algebra.

Let $\Lambda=k(Q, R)$ be a bound quiver algebra, where $k$ is a fixed field. Ringel, in [13], describes some algorithms that give a way to control the category $\Lambda$-mod when, for example, we delete certain vertices or arrows of $Q$. It allows us to analyse $\Lambda$-mod by simpler quivers whose associated bound quiver algebra may have some suitable properties. We will use two of those algorithms, namely AL.1: deleting for vertices and AL.2: deleting for arrows (see [13]). These processes allow us to obtain a simpler quiver with relations $\left(Q^{\prime}, R^{\prime}\right)$, such that $\left(Q^{\prime}, R^{\prime}\right)$-mod is a full subcategory of $(Q, R)$-mod. Furthermore, they can be applied repeatedly and alternately any given number of times.

Proposition 3.1. Let $\Lambda$ be a connected, basic k-algebra. If $\Lambda$-ind is determined by composition factors, then the quiver with relations $\left(Q_{\Lambda}, R_{\Lambda}\right)$ such that $\Lambda=$ $k\left(Q_{\Lambda}, R_{\Lambda}\right)$ satisfies the following conditions:

(i) $Q_{\Lambda}$ has no multiple arrow or subquivers $\tilde{A}_{n}$ without relations, where $n \geq 3$;

(ii) $Q_{\Lambda}$ has no subquiver $Q^{\prime}:{ }^{\alpha} Q_{a} \stackrel{\beta}{\longrightarrow} \dot{b}_{b}$ (or ${ }^{\alpha^{\prime}} \bigcirc_{a} \stackrel{\beta^{\prime}}{{ }_{b}}$ ), with $\beta \alpha \notin R_{\Lambda}$ (or $\alpha^{\prime} \beta^{\prime} \notin R_{\Lambda}$ );

(iii) $k Q_{\Lambda}$ has no oriented cycle $\mu=\alpha_{r} \alpha_{r-1} \ldots \alpha_{2} \alpha_{1}$, with $r \geq 2, \alpha_{i} \neq \alpha_{j}$ if $i \neq j$, such that $\mu \notin R_{\Lambda}$ and every subpath of $\mu$ is not a summand of a generator of $R_{\Lambda}$.

Proof. Since $\Lambda$-ind is determined by composition factors, by remark 1.1, it follows that (i) is satisfied.

(ii) Suppose that $Q_{\Lambda}$ contains a subquiver $Q^{\prime}$. We can reduce to the case that $Q_{\Lambda}=Q^{\prime}$. In fact, if $Q^{\prime}$ is a proper subquiver of $Q_{\Lambda}$, deleting the vertices $x \notin$ $\{a, b\}$ and deleting the arrows $\gamma \notin\{\alpha, \beta\}$ (or $\gamma \notin\left\{\alpha^{\prime}, \beta^{\prime}\right\}$ ), applying AL.1 and AL.2 repeatedly and alternately, we obtain the algebra $\Lambda^{\prime}=k\left(Q^{\prime}, R^{\prime}\right)$, where $\beta \alpha \notin R^{\prime}$ (or $\alpha^{\prime} \beta^{\prime} \notin R^{\prime}$ ), whose $\left(Q^{\prime}, R^{\prime}\right.$ )-mod is a full subcategory of $\Lambda$-mod. We will write the proof of the first case only, since the other one follows similarly.

Since $R_{\Lambda}$ is an admissible ideal of $k Q_{\Lambda}$ and $\beta \alpha \notin R_{\Lambda}$, there are $r$ and $s$ in $\mathbb{Z}$ greater than or equal to 2 such that $\alpha^{r}$ and $\beta \alpha^{s}$ are in $R_{\Lambda}$. Without loss of generality we can suppose that $r=s=2$. Let us consider the indecomposable projective $P_{a}=\Lambda\left(e_{a}+R\right)$, where $e_{a}$ is the trivial path of $k Q_{\Lambda}$ with origin at $a$. Then $\operatorname{rad} P_{a}=\Lambda(\alpha+R) \oplus \Lambda(\beta+R)$ with $\Lambda(\beta+R)=k(\beta+R) \cong S_{b}$. Therefore $\left[P_{a}\right]=$ $2\left[S_{a}\right]+2\left[S_{b}\right]$ in $K_{0}(\Lambda)$, and from this it follows that the module $M=P_{a} / \mathrm{rad}^{2} P_{a}$ is indecomposable and such that $[M]=2\left[S_{a}\right]+\left[S_{b}\right]$ and $\operatorname{soc} M=S_{a} \oplus S_{b}$. Let us consider the indecomposable injective $I_{b}=D P_{b}^{o p}$. Since $\Lambda^{o p}=k\left(Q^{o p}, R^{o p}\right)$, it is easy to see that $\left[I_{b}\right]=\left[D P_{b}^{o p}\right]=2\left[S_{a}\right]+\left[S_{b}\right]$ in $K_{0}\left(\Lambda^{o p}\right)$ and $\operatorname{soc} I_{b}=S_{b}$. Hence we obtain two indecomposable modules, which are the modules $M$ and $I_{b}$, having the same composition factors, but not isomorphic, which contradicts the assumption.

(iii) Suppose that there exists in $k Q_{\Lambda}$ an oriented cycle $\mu$ as in (iii). Denote by $Q^{\prime \prime}$ the subquiver of $Q_{\Lambda}$ which is the cycle $\mu$. Applying AL.1 and AL.2 to delete 
conveniently certain vertices and arrows, we obtain an algebra $\Lambda^{\prime}=k\left(Q^{\prime \prime}, R^{\prime \prime}\right)$ such that $u \notin R^{\prime \prime}$ and whose $\left(Q^{\prime \prime}, R^{\prime \prime}\right)$-mod is a full subcategory of $\Lambda$-mod. But $\Lambda^{\prime}=k\left(Q^{\prime \prime}, R^{\prime \prime}\right)$ is a connected Nakayama algebra such that the rank of $K_{0}\left(\Lambda^{\prime}\right)$ is $r \geq 2$. Since $\mu \notin R^{\prime \prime}$, it follows that there is an indecomposable projective $\Lambda^{\prime}$-module $P$ with $l(P)>r$ (for example, $\left.P=P_{o\left(\alpha_{1}\right)}\right)$. So, by proposition $2.1, \Lambda^{\prime}$-ind is not determined by composition factors, and as a consequence $\Lambda$-ind is not determined by composition factors, which is a contradiction to the assumption on $\Lambda$-ind.

To conclude this section, we present an example of an algebra $\Lambda$ whose $\Lambda$-ind is multiplicity-free but is not determined by composition factors, which shows that the property (ii) does not imply (i).

Example 3.1. Let $\Lambda=k(Q, R)$, where $Q$ is the quiver below and $R$ is given by $\gamma \beta \alpha=0$ and $\alpha \gamma=0$.

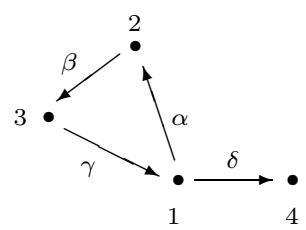

$\Lambda$ is a representation-finite biserial algebra, where $\operatorname{dim}_{k} \Lambda=12$, such that all indecomposable projective modules, except $P_{1}$, and all indecomposable injective modules are uniserial $\left(P_{1}\right.$ is not uniserial, since $\operatorname{rad} P_{1}=P_{4} \oplus N$, where $N \cong$ $\left.P_{2} / \mathrm{rad}^{2} P_{2}\right)$. The Auslander-Reiten quiver of $\Lambda$, illustrated below, shows that $\Lambda$ ind is multiplicity-free. But $\Lambda$-ind is not determined by composition factors, since, for example, $P_{1}$ and $P_{2}$ are nonisomorphic modules having the same composition factors.

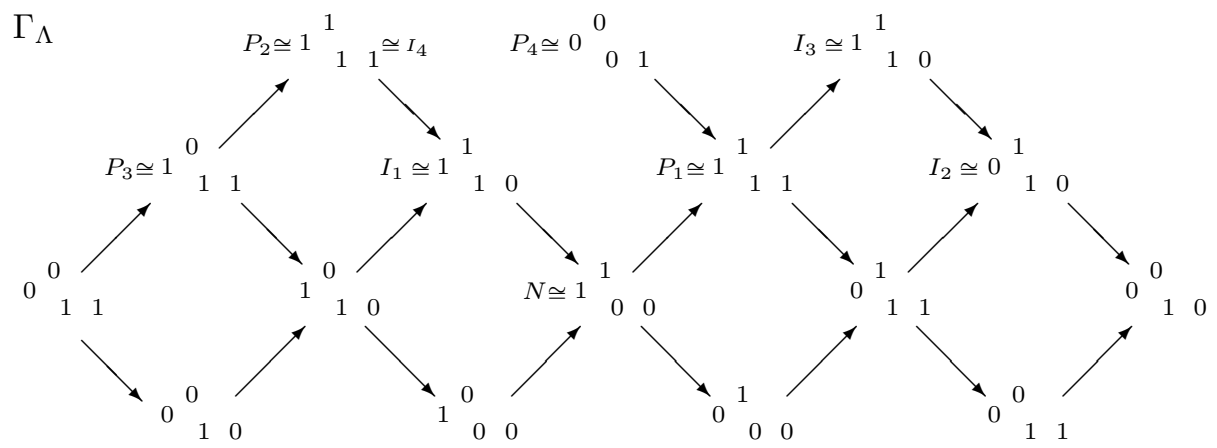

\section{IndeComposable EXtensions of A SIMPLE MOdULE}

In [12], Pogorzaly and Skowroński settled the diagrammatic structure of the representation-finite biserial algebras, through the following theorem.

Theorem 4.1. (see [12], th.2) Any representation-finite biserial k-algebra $\Lambda$ is isomorphic to a bound quiver algebra $k\left(Q_{\Lambda}, R_{\Lambda}\right)$, where $\left(Q_{\Lambda}, R_{\Lambda}\right)$ satisfies the following conditions, denoted by (SP): 
(1) The number of arrows starting (ending) at any fixed vertex of $Q_{\Lambda}$ is at most two.

(2) For any arrow $\alpha$ of $Q_{\Lambda}$ there is at most one arrow $\beta$ and at most one arrow $\gamma$ such that $\beta \alpha$ and $\alpha \gamma$ are not in $R_{\Lambda}$.

(3) There is an upper bound for the length of paths in $Q_{\Lambda}$ which are not in $R_{\Lambda}$. $Q_{\Lambda}$.

(4) $R_{\Lambda}$ is generated by paths and by the differences of pairs of parallel paths of

From now on, when we refer to a representation-finite biserial $k$-algebra $\Lambda$, $\left(Q_{\Lambda}, R_{\Lambda}\right)$ will denote a fixed bound quiver satisfying the conditions (SP) of the above theorem.

Remark 4.1. In a bound quiver $(Q, R)$ which satisfies the above conditions (SP) it is easy to see that the following conditions are verified. (4.1.a) A pair of parallel paths of $k Q$, which are not in $R$, does not contain common arrows, and their vertices, except the origin and the end, are vertices in just one of these paths. (4.1.b) Given a path $\mu \in k Q-R$, no proper subpath of $\mu$ is a summand of a generator of $R$. (4.1.c) Given an arrow $\alpha: i \rightarrow j$ of $Q$, then the conditions (SP)1, 2, 3 determine the unique path not in $R$ starting in $\alpha$, which is the maximal path among all paths $\mu_{\alpha}$ which are not in $R$ and starting in $\alpha$. We denote this path by $w_{\alpha}$. Moreover, if $Q$ does not contain loops or subpaths which are oriented cycles without repeated arrows, then the arrows of $w_{\alpha}$ are all distinct and each vertex of $w_{\alpha}$, except the origin and the end, belongs to exactly two of these arrows.

To simplify the reading, we introduce some notations. Given a path $w$ of $Q$, we denote by $w_{1}$ the set of the arrows in $w$ and by $w_{0}$ the set of the vertices in $w$. The expression " $a$ path $u_{\alpha}$ " means a path $u$ starting in the arrow $\alpha$. Moreover, we denote by $\hat{u_{\alpha}}$ the oriented cycle of minimal length among all the oriented cycles (if they exist), starting in $\alpha$, which are not in $R$.

Using these notations, we state (without proof) the following technical lemma.

Lemma 4.2. Let $k(Q, R)$ be a bound quiver algebra, where $(Q, R)$ satisfies the conditions $(S P)$ of theorem 4.1 and $Q$ contains neither loops nor double arrows. Let $i$ be a fixed vertex of $Q$.

(a) Suppose that $i$ is the origin of exactly one arrow $\alpha: i \rightarrow j($ notation $i=o(\alpha)$ ).

(a.1) If the end of $w_{\alpha}$ (notation $e\left(w_{\alpha}\right)$ ) is $i$, then $w_{\alpha}=\left(\hat{u_{\alpha}}\right)^{r}$, for some $r \geq 1$.

(a.2) If $e\left(w_{\alpha}\right) \neq i$, then either (i) $w_{\alpha}=u_{\alpha}^{\prime}\left(\hat{u_{\alpha}}\right)^{r}$, for some $r \geq 1$, where $u_{\alpha}^{\prime}$ is a proper subpath of $\hat{u_{\alpha}}$, or (ii). $w_{\alpha}$ does not contain oriented cycles starting at $i$;

(b) Suppose that $i$ is the origin of two distinct arrows $\alpha: i \rightarrow j_{1} e \beta: i \rightarrow j_{2}$.

(b.1) If there are two oriented cycles, one starting in $\alpha$ and the other starting in $\beta$, then $w_{\alpha}=\hat{u_{\alpha}}$ and $w_{\beta}=\hat{u_{\beta}}$, and such oriented cycles are parallel paths (so $w_{\alpha}-w_{\beta} \in R$ ).

(b.2) If $e\left(w_{\alpha}\right)=i$ and $e\left(w_{\beta}\right) \neq i$, then $w_{\alpha}=\left(\hat{u_{\alpha}}\right)^{r}$, for some $r \geq 1$, $w_{\beta}$ does not contain oriented cycles starting at $i,\left(w_{\alpha}\right)_{0} \cap\left(w_{\beta}\right)_{0}=i$ and $\left(w_{\alpha}\right)_{1} \cap\left(w_{\beta}\right)_{1}=$ $\emptyset$.

(b.3) If $e\left(w_{\alpha}\right)=e\left(w_{\beta}\right) \neq i$, then either ( $\left.i\right)$ one of them, say $w_{\alpha}$, contains an oriented cycle starting in $\alpha$, and, in this case, $w_{\alpha}=w_{\beta} \hat{u_{\alpha}}$ and $\hat{u_{\alpha}}$ does not contain the arrow $\beta$, or (ii) $w_{\alpha}$ and $w_{\beta}$ are parallel $\left(\right.$ so, $w_{\alpha}-w_{\beta} \in R$ ).

(b.4) If $e\left(w_{\alpha}\right) \neq e\left(w_{\beta}\right)$, with $i \in\left\{e\left(w_{\alpha}\right), e\left(w_{\beta}\right)\right\}$, then either (i) $w_{\alpha}=u_{\alpha}^{\prime}\left(\hat{u_{\alpha}}\right)^{r}$, for some $r \geq 1$, where $u_{\alpha}^{\prime}$ is a proper subpath of $\hat{u_{\alpha}}, w_{\beta}$ contains no 
oriented cycle starting in $\beta,\left(w_{\alpha}\right)_{0} \cap\left(w_{\beta}\right)_{0}=i$ and $\left(w_{\alpha}\right)_{1} \cap\left(w_{\beta}\right)_{1}=\emptyset$, or (ii) $w_{\alpha}=u_{\beta} \hat{u_{\alpha}}$ and $w_{\beta}=\gamma u_{\beta}$, where $u_{\beta}$ is a proper subpath of $w_{\beta}$ starting at $e\left(w_{\alpha}\right), \gamma$ is a subpath of $w_{\beta}$ starting at $e\left(w_{\alpha}\right)$ and such that $\left(\hat{u_{\alpha}}\right)_{0} \cap\left(w_{\beta}\right)_{0}=i$, and $\left(\hat{u_{\alpha}}\right)_{1} \cap\left(w_{\beta}\right)_{1}=\emptyset$ or (iii) $w_{\alpha}$ and $w_{\beta}$ contain no oriented cycle starting at $i$, and $i$ is the unique common vertex among them and they do not have common arrows.

The structure given by theorem 4.1, and the description in lemma 4.2 and proposition 3.1, allow us initially to prove the following theorem, where we shall use the notations above.

Theorem 4.3. Let $\Lambda$ be a biserial $k$-algebra whose ordinary quiver $Q_{\Lambda}$ does not contain any loop. Suppose that $\Lambda$-ind is determined by composition factors. Then each projective indecomposable $\Lambda$-module and each injective indecomposable $\Lambda$ module is multiplicity-free.

Proof. Using the duality $D$, it is enough to show that the indecomposable projective modules are multiplicity-free. By remark 1.1, $\Lambda$ is representation-finite and, being a biserial algebra, by theorem 4.1 it is isomorphic to $k\left(Q_{\Lambda}, R_{\Lambda}\right)$, where $\left(Q_{\Lambda}, R_{\Lambda}\right)$ satisfies the conditions (SP) and the conditions of proposition 3.1.

Suppose that there is a projective indecomposable module $P$ and a simple module $S$ such that $S$ is a composition factor of $P$ with multiplicity greater than or equal to 2. So, $P \cong P_{i}$ for some vertex $i$ of $Q_{\Lambda}, S \cong S_{j}$ for some vertex $j$ of $Q_{\Lambda}$ and $\operatorname{dim}_{k} P(j) \geq 2$.

$1^{\underline{s t}}$ case: $S \cong S_{i}$ (i.e, $i=j$ ). Then there exists an oriented cycle in $k Q_{\Lambda}-R_{\Lambda}$, starting at $i$. Depending on whether $i$ is the origin of one arrow or of two distinct arrows, we have one of the cases (a.1), (a.2.i), (b.1), (b.2), (b.3.i), (b.4.i) or (b.4.ii) in lemma 4.2. If we have case (b.1), then the maximal paths $w_{\alpha}$ and $w_{\beta}$ are parallel and $w_{\alpha}-w_{\beta} \in R_{\Lambda}$. Hence,

$\operatorname{rad} P_{i}=\Lambda\left(\alpha+R_{\Lambda}\right)+\Lambda\left(\beta+R_{\Lambda}\right) \quad$ and $\quad \operatorname{soc} P_{i}=k\left(w_{\alpha}+R_{\Lambda}\right)=k\left(w_{\beta}+R_{\Lambda}\right) \cong S_{i}$.

So the indecomposable modules $M=P_{i} / \Lambda\left(\beta+R_{\Lambda}\right)$ and $N=\Lambda\left(\alpha+R_{\Lambda}\right)$ have the same composition factors (the simple modules $S_{x}$, where $\left.x \in\left(w_{\alpha}\right)_{0}\right)$, but they are not isomorphic, since top $M \cong S_{i}$ and top $N \cong S_{e(\alpha)}$. This is a contradiction to $\Lambda$ ind being determined by composition factors. In any of the cases (a.1), (a.2.i), (b.2), (b.3.i), (b.4.i) or (b.4.ii), using the notation of the last lemma, we can consider the oriented cycle $\hat{u_{\alpha}}$. By the minimality of the length of $\hat{u_{\alpha}}$, it contains no repeated arrows and, by remark 4.1.b, this cycle contains no subpath which is a summand of a generator of $R_{\Lambda}$. Then we get a contradiction to the conditions on $\left(Q_{\Lambda}, R_{\Lambda}\right)$. $2^{\frac{n d}{d}}$ case: $S \neq S_{i}$ (i.e, $i \neq j$ ). In this case, there is no oriented cycle in $Q_{\Lambda}$ starting at $i$ which is not in $R_{\Lambda}$. Then, from the condition (SP) 4 and from $\operatorname{dim}_{k} P(j)=$ $\operatorname{dim}_{k} P_{i}(j) \geq 2$, it follows that there is an oriented cycle $\mu \notin R_{\Lambda}$, starting at $j$, such that $i$ is not a vertex of $\mu$. So, relative to the paths with origin at $i$, we have one of the remaining cases of lemma 4.2, that is, (a.2.ii), (b.3.ii) or (b.4.ii). In case (a.2.ii), $\mu$ is a proper subpath of $w_{\alpha}$ and $j$ is the end of two arrows $\gamma$ and $\gamma^{\prime}$, with $\gamma^{\prime}$ in $\mu$, and $j$ is the origin of at least one arrow $\delta^{\prime}$, with $\delta^{\prime}$ in $\mu$, such that $\mu \gamma \notin R_{\Lambda}$ and $\delta^{\prime} \gamma^{\prime} \in R_{\Lambda}$ (if there is another arrow $\delta$ in $w_{\alpha}$, starting at $j$, then $\delta \mu \gamma \notin R_{\Lambda}$ and $\left.\delta \gamma \in R_{\Lambda}\right)$. From this, it follows that $\mu$ is the unique oriented cycle starting at $j$, and so $\mu=\hat{u_{\delta^{\prime}}}$ (since $\delta^{\prime} \gamma^{\prime} \in R_{\Lambda}$ ). In case (b.3.ii), $\mu$ is a proper subpath of exactly one of the parallel paths $w_{\alpha}$ and $w_{\beta}$, let us say $w_{\alpha}$, and $j$ is not the end of any of them. Therefore, $j$ is the end of two distinct arrows $\gamma$ and $\gamma^{\prime}$ of $w_{\alpha}$ and the origin 
of two distinct arrows $\delta$ and $\delta^{\prime}$ as in (a.2.ii), and hence, $\mu=\hat{u_{\delta^{\prime}}}$. If we have case (b.4.ii), since $\left(w_{\alpha}\right)_{1} \cap\left(w_{\beta}\right)_{1}=\emptyset$ and $\left.\left(w_{\alpha}\right)_{0} \cap\left(w_{\beta}\right)_{0}\right)=\{i\}$, we infer that $\mu$ is a proper subpath of only one of them, and relative to the arrows which have vertices at $j$ it behaves as in case (a.2.i). Hence, in any of these possibilities, as in the first case, $Q_{\Lambda}$ contains an oriented cycle, not in $R_{\Lambda}$, whose arrows are all different and such that no subpath of it is a summand of a generator of $R_{\Lambda}$, which gives a contradiction to condition (iii) of proposition 3.1. So, any projective indecomposable $\Lambda$-module is multiplicity-free.

Remark 4.2. In fact, the proof of theorem 4.3 follows from the fact that any oriented cycle of $k Q_{\Lambda}$ is in $R_{\Lambda}$. Therefore if in this theorem we require that all oriented cycles are in the relation set instead of $\Lambda$-ind being determined by composition factors, its statement is still true.

To attain our aim, we need some preliminaries. Given a simple module $S$, we denote by $i_{S}$ the vertex of $Q_{\wedge}$ which is associated to $S$. We start with another technical lemma.

Lemma 4.4. Let $\Lambda$ be a representation-finite biserial algebra whose ordinary quiver $Q_{\Lambda}$ has no loop and such that all oriented cycles are in $R_{\Lambda}$. Let $N$ be a $\Lambda$-module, with $l(N) \geq 2$, and let $v \in N$ be such that the simple $\Lambda$-module $S=k v \subset N$ is not a direct summand of $N$. Then

(i) There is a path

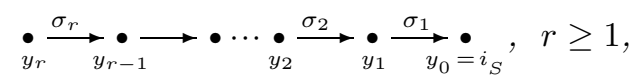

and a family $\left(v_{i}\right)_{i} \in \bigoplus_{i=0}^{r} N\left(y_{i}\right)$, with $v_{0}=v$, such that $N\left(y_{i}\right)=k v_{i} \oplus N^{\prime}\left(y_{i}\right)$, $N\left(\sigma_{i}\right)\left(v_{i}\right)=v_{i-1}$ and $N\left(\sigma_{i}\right)\left(N^{\prime}\left(y_{i}\right)\right) \subset N^{\prime}\left(y_{i-1}\right)$, for $1 \leq i \leq r$, and $\operatorname{Im} N(\delta) \subset$ $N^{\prime}\left(y_{r}\right)$, for any arrow $\delta$ ending at $y_{r}$.

(ii) If there are two arrows $\sigma: y \rightarrow i_{S}$ and $\bar{\sigma}: \bar{y} \rightarrow i_{S}$, with $\sigma \neq \bar{\sigma}$, such that $v \in \operatorname{Im} N(\sigma) \cap \operatorname{Im} N(\bar{\sigma})$, then the quiver $Q_{N}$ of $N$ contains the subquiver

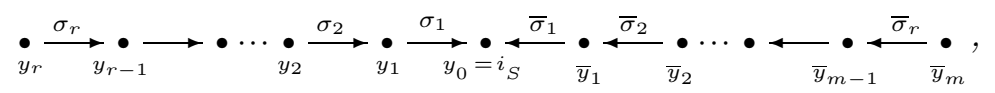

with $r, m \geq 1, \sigma_{1}=\sigma, \bar{\sigma}_{1}=\bar{\sigma}$. For this subquiver there are the families

$$
\left(v_{i}\right)_{i} \in \bigoplus_{i=0}^{r} N\left(y_{i}\right) \text { and }\left(\bar{v}_{j}\right)_{j} \in \bigoplus_{j=0}^{m} N\left(\bar{y}_{j}\right),
$$

where $\bar{v}_{0}=v=v_{0}$, such that $N\left(y_{i}\right)=k v_{i} \oplus N^{\prime}\left(y_{i}\right), N\left(\sigma_{i}\right)\left(v_{i}\right)=v_{i-1}, N\left(\sigma_{i}\right)\left(N^{\prime}\left(y_{i}\right)\right) \subset$ $N^{\prime}\left(y_{i-1}\right), 1 \leq i \leq r, N\left(\bar{y}_{i}\right)=k \bar{v}_{i} \oplus N^{\prime}\left(\bar{y}_{i}\right), N\left(\bar{\sigma}_{i}\right)\left(\bar{v}_{i}\right)=\bar{v}_{i-1}, N\left(\bar{\sigma}_{i}\right)\left(N^{\prime}\left(\bar{y}_{i}\right)\right) \subset$ $N^{\prime}\left(\bar{y}_{i-1}\right), 1 \leq i \leq m$. Furthermore, $\operatorname{Im} N(\delta) \subset N^{\prime}\left(y_{r}\right)$, for all arrows $\delta$ ending at $y_{r}$, and $\operatorname{Im} N(\bar{\delta}) \subset N^{\prime}\left(\bar{y}_{m}\right)$, for all arrows $\bar{\delta}$ ending at $\bar{y}_{m}$.

Proof. It follows, from remark 4.2 , that each injective indecomposable $\Lambda$-module $I$ is of type $A_{n}$ or a projective-injective non-uniserial. Therefore, $I(x) \cong k$ for all $x \in \operatorname{supp} I$, and $I(\alpha) \cong \mathbb{1}$ for every arrow $\alpha$ of the quiver $Q_{I}$.

We consider $N\left(i_{S}\right)=k v \oplus N^{\prime}\left(i_{S}\right)$. Let $\iota: S \hookrightarrow N$ be the inclusion of $S=k v$ in $N$ and $\jmath: S \hookrightarrow I_{S}$ be the injective envelope of $S$. Then, there exists a morphism of $k$-representations $\lambda=\left(\lambda_{x}\right)_{x}: N \rightarrow I_{S}$ such that $\lambda \circ \iota=\jmath$. Since $I_{S}\left(i_{S}\right)=k v$, we have that $\operatorname{ker} \lambda_{i_{S}}=N^{\prime}\left(i_{S}\right)$. So, since $S$ is not a direct summand of $N, L=\operatorname{Im} \lambda$ is a submodule of $I_{S}$ containing $S$ properly, and hence it is an indecomposable module 
with $l(L) \geq 2$. Since $I_{S}$ is of type $A_{n}$ or a projective-injective non-uniserial, there is a subpath $\mu: y_{r} \stackrel{\sigma_{r}}{\rightarrow} y_{r-1} \ldots y_{2} \stackrel{\sigma_{2}}{\rightarrow} y_{1}=y \stackrel{\sigma_{1}}{\rightarrow} y_{0}=i_{S}, r \geq 1$, in the quiver of $L$, such that $\left(\operatorname{Im} \lambda_{y_{i}}\right)\left(\sigma_{i}\right) \cong \mathbb{1}$ for $0 \leq i \leq r$, and $\lambda_{x}=0$ for each vertex $x=o(\delta)$, for every arrow $\delta$, where $e(\delta)=y_{r}$. It means that $\mu$ is the path of maximal length of $Q_{L}$ ending at $\sigma_{1}$ with the property $L(\mu) \cong \mathbb{1}$. Moreover, for each $i=1,2, \ldots, r$, since $\lambda_{y_{i}} \neq 0$, the following diagram is commutative:

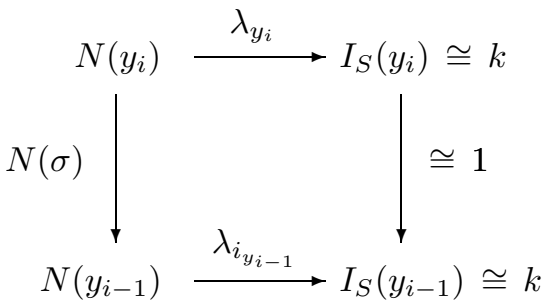

From the commutativity of the above diagram and from $L \cong N / k e r \lambda$, we infer that we can choose a family $\left(v_{i}\right)_{i} \in \bigoplus_{i=0}^{r} N\left(y_{i}\right)$, where $v_{0}=v$, such that $N\left(y_{i}\right)=k v_{i} \oplus N^{\prime}\left(y_{i}\right)$, where $N^{\prime}\left(y_{i}\right)=k e r \lambda_{y_{i}}$, and such that $N\left(\sigma_{i}\right)\left(v_{i}\right)=v_{i-1}$ and $N\left(\sigma_{i}\right)\left(N^{\prime}\left(y_{i}\right)\right) \subset N^{\prime}\left(y_{i-1}\right)$, for $1 \leq i \leq r$. Moreover, it follows easily that $\operatorname{Im} N(\delta) \subset \operatorname{ker} \lambda_{y_{r}}=N^{\prime}\left(y_{r}\right)$, for each $\delta$ of $Q_{N}$, where $e(\delta)=y_{r}$ (since $\lambda_{x}=0$, where $x=o(\delta))$. So, (i) is proved.

Let $\sigma: y \rightarrow i_{S}$ and $\bar{\sigma}: \bar{y} \rightarrow i_{S}$ be the two arrows of $Q_{\Lambda}$, with $\sigma \neq \bar{\sigma}$, such that $v \in \operatorname{Im} N(\sigma) \cap \operatorname{Im} N(\bar{\sigma})$. Since $Q_{\Lambda}$ does not have loops or double arrows, then $\bar{y} \notin\left\{y, i_{S}\right\}$. In this case, $I_{S}$ is a non-uniserial module, and it follows from this that $l(L) \geq 3$ and that $\lambda_{y}$ and $\lambda_{\bar{y}}$ are not zero. So, $L$ is a non-uniserial submodule of $I_{S}$, whose quiver is $y_{r} \stackrel{\sigma_{r}}{\rightarrow} y_{r-1} \ldots y_{2} \stackrel{\sigma_{2}}{\rightarrow} y_{1}=y \stackrel{\sigma_{1}}{\rightarrow} y_{0}=i_{S} \stackrel{\bar{\sigma}_{1}}{\longleftarrow} \bar{y}_{1}=\bar{y} \stackrel{\bar{\sigma}_{2}}{\longleftarrow} \bar{y}_{2} \ldots \bar{y}_{m-1} \stackrel{\bar{\sigma}_{m}}{\leftarrow} \bar{y}_{m}$, where $r, m \geq 1, \sigma_{1}=\sigma, \bar{\sigma}_{1}=\bar{\sigma}$. (We observe that, if $\bar{y}_{m}=y_{r}$, then $I_{S}$ is an injectiveprojective non-uniserial and $\lambda$ is an epimorphism). The rest of the proof follows using arguments as in part (i) on each of the two paths ending at $i_{S}$, which make $Q_{L}$.

Remark 4.3. Under the hypothesis of lemma 4.4, if there are two different arrows $\sigma: y \rightarrow i_{S}$ and $\bar{\sigma}: \bar{y} \rightarrow i_{S}$ of $Q_{\Lambda}$ such that $v \in \operatorname{Im} N(\sigma)$ but $v \notin \operatorname{Im} N(\bar{\sigma})$, then, by a conveniently chosen basis for $N(\bar{y})$, we infer that $\operatorname{Im} N(\bar{\sigma}) \subset N^{\prime}\left(i_{S}\right)$. Furthermore, using (SP)2, we can describe the morphism $N(\alpha)$, for each arrow $\alpha$ different from $\sigma_{i}$, for $1 \leq i \leq r$, having one of its vertices at $y_{i}, 0 \leq i \leq r$. If $\alpha \neq \sigma_{i}, 1 \leq i \leq r-1$, with $o(\alpha) \in\left\{y_{i}, i_{S}\right\}$, then $N(\alpha)\left(v_{i}\right)=0$ for $1 \leq i \leq r$; if $\alpha \neq \sigma_{i+1}, 1 \leq i \leq r-1$, with $e(\alpha)=y_{i}$, then $\operatorname{Im} N(\alpha) \subset N^{\prime}\left(y_{i}\right)$. Even in the case that $v \in \operatorname{Im} N(\sigma) \cap \operatorname{Im} N(\bar{\sigma})$, we can also describe similarly the morphisms $N(\gamma)$, for each $\gamma \neq \bar{\sigma}_{j}, 1 \leq j \leq m$, and having one of its vertices at $\bar{y}_{j}$, for $1 \leq j \leq m-1$.

The last lemma and remark 4.3 allow us to determine if a given module $M$ is indecomposable or not through of the number of arrows ending at the vertices of the quiver of $M$, which are associated to their simple submodules.

Theorem 4.5. Let $\Lambda=k\left(Q_{\Lambda}, R_{\Lambda}\right)$ be a representation-finite biserial algebra whose ordinary quiver $Q_{\Lambda}$ has no loop and such that all oriented cycles of $Q_{\Lambda}$ are in $R_{\Lambda}$. Let $M$ be a $\Lambda$-module such that $M=N+X$, with $l(N), l(X) \geq 2$, and suppose that there exists $0 \neq v \in M$ such that $N \cap X=k v=S$. If there is an arrow $\sigma$ ending at $i_{S}$ such that $v \in \operatorname{Im} N(\sigma) \cap \operatorname{Im} X(\sigma)$, then $M$ is decomposable. 
Proof. Since $S=k v \subset M$, it follows that $M(\alpha)(v)=0$ for each arrow $\alpha$ where $o(\alpha)=i_{S}$. On the other hand, setting $N\left(i_{S}\right)=k v \oplus N^{\prime}\left(i_{S}\right)$ and $X\left(i_{S}\right)=k v \oplus X^{\prime}\left(i_{S}\right)$, we can rewrite $M=\left(M(x)_{x}, M(\alpha)_{\alpha}\right)$ in the following way:

$$
\begin{gathered}
M(x)= \begin{cases}N(x) \oplus X(x) & \text { if } x \neq i_{S}, \\
k v \oplus N^{\prime}\left(i_{S}\right) \oplus X^{\prime}\left(i_{S}\right) & \text { if } x=i_{S},\end{cases} \\
M(\alpha)= \begin{cases}N(\alpha) \oplus X(\alpha) & \text { if } i_{S} \notin\{o(\alpha), e(\alpha)\}, \\
N(\alpha)+X(\alpha) & \text { if } e(\alpha)=i_{S}, \\
N^{\prime}(\alpha) \oplus X^{\prime}(\alpha) & \text { if } o(\alpha)=i_{S},\end{cases}
\end{gathered}
$$

where $X^{\prime}(\alpha)$ and $N^{\prime}(\alpha)$ denote, respectively, the restriction of $X(\alpha)$ to the subspace $X(o(\alpha))$ and of $N(\alpha)$ to the subspace $N(o(\alpha))$. It is clear that $S$ is neither a direct summand of $N$ nor of $X$. We consider several cases.

Let us assume first that, besides the arrow $\sigma: y \rightarrow i_{S}$, there is in $Q_{M}$ another arrow $\bar{\sigma}: \bar{y} \rightarrow i_{S}$, where $\bar{\sigma} \neq \sigma$ (hence $y \neq \bar{y}$ ) and that $v \in \operatorname{Im} N(\bar{\sigma}) \cap \operatorname{Im} X(\bar{\sigma})$. Applying lemma 4.4(ii) to $N$ and $X$, we see that there is a subquiver $\mu_{N}$ of $Q_{N}$, where $\mu_{N}: y_{r} \stackrel{\sigma_{r}}{\rightarrow} \ldots \rightarrow y_{s} \stackrel{\sigma_{s}}{\rightarrow} \ldots \rightarrow y_{2} \stackrel{\sigma_{2}}{\rightarrow} y_{1}=y \stackrel{\sigma_{1}}{\rightarrow} y_{0}=i_{S} \stackrel{\bar{\sigma}_{1}}{\leftarrow} \bar{y}_{1}=\bar{y} \stackrel{\bar{\sigma}_{2}}{\longleftarrow} \bar{y}_{2} \leftarrow$ $\ldots \stackrel{\bar{\sigma}_{m}}{\leftarrow} \bar{y}_{m}$, and there is a subquiver $\mu_{X}$ of $Q_{X}$, where $\mu_{X}: y_{s} \stackrel{\sigma_{s}}{\rightarrow} \ldots \rightarrow y_{2} \stackrel{\sigma_{2}}{\rightarrow}$ $y_{1}=y \stackrel{\sigma_{1}}{\rightarrow} y_{0}=i_{S} \stackrel{\bar{\sigma}_{1}}{\longleftarrow} \bar{y}_{1}=\bar{y} \stackrel{\bar{\sigma}_{2}}{\longleftarrow} \bar{y}_{2} \leftarrow \ldots \leftarrow \bar{y}_{q-1} \stackrel{\bar{\sigma}_{q}}{\longleftarrow} \bar{y}_{q}$, where $1 \leq s \leq r$, and $q, m \geq 1, \sigma_{1}=\sigma, \bar{\sigma}_{1}=\bar{\sigma}$. For these subquivers, also by lemma 4.4(ii), there are families $\left(v_{i}\right)_{i} \in \bigoplus_{i=0}^{r} N\left(y_{i}\right),\left(\bar{v}_{i}\right)_{i} \in \bigoplus_{i=0}^{m} N\left(\bar{y}_{i}\right)$, and $\left(w_{j}\right)_{j} \in \bigoplus_{j=0}^{s} X\left(y_{j}\right)$ and $\left(\bar{w}_{j}\right)_{j} \in \bigoplus_{j=0}^{q}, X\left(\bar{y}_{j}\right)$, where $v_{0}=w_{0}=\bar{v}_{0}=\bar{w}_{0}=v$, such that $N\left(y_{i}\right)=k v_{i} \oplus N^{\prime}\left(y_{i}\right)$, for $0 \leq i \leq r, N\left(\bar{y}_{i}\right)=k \bar{v}_{i} \oplus N^{\prime}\left(\bar{y}_{i}\right)$, for $0 \leq i \leq m$, and $X\left(y_{j}\right)=k w_{j} \oplus X^{\prime}\left(y_{j}\right)$, for $0 \leq j \leq s$ and $X\left(\bar{y}_{j}\right)=k \bar{w}_{j} \oplus X^{\prime}\left(\bar{y}_{j}\right)$, for $0 \leq j \leq q$. Moreover, the morphisms $X(\alpha)$ and $N(\alpha)$ are as in lemma 4.4 and in remark 4.3, for the arrows $\alpha$ starting (ending) at $y_{i}$ and $\bar{y}_{j}$.

Since all the paths of $k Q_{\Lambda}-R_{\Lambda}$ ending in $\bar{\sigma}$ are ordered by their lengths, it follows that $q \leq m$ or $m \leq q$. Suppose now that $1 \leq m \leq q$. Using the previous

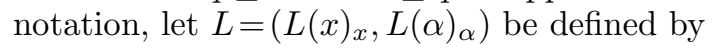

$$
\begin{gathered}
L(x)= \begin{cases}k v_{i} \oplus N^{\prime}\left(y_{i}\right) \oplus X^{\prime}\left(y_{i}\right) & \text { if } x=y_{i}, \text { for } 0 \leq i \leq s, \\
k v_{i} \oplus N^{\prime}\left(y_{i}\right) \oplus X\left(y_{i}\right) & \text { if } x=y_{i}, \text { for } s+1 \leq i \leq r, \\
N^{\prime}\left(\bar{y}_{j}\right) \oplus k \bar{w}_{j} \oplus X^{\prime}\left(\bar{y}_{j}\right) & \text { if } x=\bar{y}_{j}, \text { for } 1 \leq j \leq m, \\
N\left(\bar{y}_{j}\right) \oplus k \bar{w}_{j} \oplus X^{\prime}\left(\bar{y}_{j}\right) & \text { if } x=\bar{y}_{j}, \text { for } m+1 \leq j \leq q, \\
N(x) \oplus X(x) & \text { for the other vertices, }\end{cases} \\
L(\alpha)= \begin{cases}N\left(\sigma_{i}\right) \oplus X^{\prime}\left(\sigma_{i}\right) & \text { if } \alpha=\sigma_{i}, \text { for } 1 \leq i \leq s, \\
N^{\prime}\left(\bar{\sigma}_{i}\right) \oplus X\left(\bar{\sigma}_{i}\right) & \text { if } \alpha=\bar{\sigma}_{i}, \text { for } 1 \leq i \leq m, \\
N(\alpha) \oplus X(\alpha) & \text { for the other arrows. }\end{cases}
\end{gathered}
$$

It is easy to verify that $L$ is a representation of $\left(Q_{\Lambda}, R_{\Lambda}\right)$. Let $\imath=\left(\imath_{x}\right)_{x}: L \rightarrow M$ be a family where, for each $x, \imath_{x}: L(x) \rightarrow M(x)$ is the natural inclusion from the space $L(x)$ to the space $M(x)$. It is also easy to prove that it admits a left inverse morphism $p=\left(p_{x}\right)_{x}: M \rightarrow L$. The components $p_{x}$ of $p$ are the following. For $1 \leq i \leq s, p_{y_{i}}: k v_{i} \oplus N^{\prime}\left(y_{i}\right) \oplus k w_{i} \oplus X^{\prime}\left(y_{i}\right) \rightarrow k v_{i} \oplus N^{\prime}\left(y_{i}\right) \oplus X^{\prime}\left(y_{i}\right)$ is defined by $p_{y_{i}}\left(a v_{i}+n^{\prime}+b w_{i}+x^{\prime}\right)=(a+b) v_{i}+n^{\prime}+x^{\prime}$, with $a, b \in k, x^{\prime} \in X^{\prime}\left(y_{i}\right)$ and $n^{\prime} \in N^{\prime}\left(y_{i}\right)$; for $1 \leq i \leq m, p_{\bar{y}_{i}}: k \bar{v}_{i} \oplus N^{\prime}\left(\bar{y}_{i}\right) \oplus k \bar{w}_{i} \oplus X^{\prime}\left(\bar{y}_{i}\right) \rightarrow N^{\prime}\left(\bar{y}_{i}\right) \oplus k \bar{w}_{i} \oplus X^{\prime}\left(\bar{y}_{i}\right)$ is defined by $p_{\bar{y}_{i}}\left(a \bar{v}_{i}+n^{\prime}+b \bar{w}_{i}+x^{\prime}\right)=(a+b) \bar{w}_{i}+n^{\prime}+x^{\prime}$, with $a, b \in k, n^{\prime} \in N^{\prime}\left(\bar{y}_{i}\right)$ and $x^{\prime} \in X^{\prime}\left(\bar{y}_{i}\right)$ and, for the remaining vertices $x, p_{x}: N(x) \oplus X(x) \rightarrow N(x) \oplus X(x)$ is the identity. We leave the verification to the reader. 
For the remaining cases (that is, either $v \in \operatorname{Im} N(\bar{\sigma}) \cap \operatorname{Im} X(\bar{\sigma})$ and $q \leq m$, or $v \notin \operatorname{Im} N(\bar{\sigma}) \cap \operatorname{Im} X(\bar{\sigma})$, or $\sigma$ is the unique arrow of $Q_{M}$ ending at $i_{S}$ such that $v \in \operatorname{Im} N(\sigma) \cap \operatorname{Im} X(\sigma))$, by the application of lemma 4.4 and remark 4.3 and a slight modification of the above arguments, it is not difficult to verify that $M=N \oplus X / k v$. So the theorem is proved.

Corollary 4.6. Let $\Lambda=k\left(Q_{\Lambda}, R_{\Lambda}\right)$ be a representation-finite biserial $k$-algebra whose ordinary quiver $Q_{\Lambda}$ does not have loops and such that all oriented cycles of $Q_{\Lambda}$ are in $R_{\Lambda}$. Let $M$ be a $\Lambda$-module such that $M=N+X$, where $l(N), l(X) \geq 2$ and $N \cap X=S=k v$, for some $0 \neq v \in M$. If $M$ is indecomposable, then there is a unique arrow $\sigma: y \rightarrow i_{S}$ such that $v \in \operatorname{Im} N(\sigma)$, and a unique arrow $\bar{\sigma}: \bar{y} \rightarrow i_{S}$, where $\sigma \neq \bar{\sigma}$, such that $v \in \operatorname{Im} X(\bar{\sigma})$.

Proof. The proof follows immediately from the fact that $M$ is indecomposable and from lemma 4.4 and theorem 4.5 .

Theorem 4.7. Let $\Lambda=k\left(Q_{\Lambda}, R_{\Lambda}\right)$ be a representation-finite biserial algebra whose ordinary quiver $Q_{\Lambda}$ has no loop and such that all oriented cycles of $k Q_{\Lambda}$ are in $R_{\Lambda}$. Let $M$ be a $\Lambda$-module and $S$ be a simple $\Lambda$-module such that $S \subset M$ and $M / S=M_{1} \oplus M_{2} \oplus \ldots \oplus M_{t}$, where $t \geq 1$ and each $M_{j}$ is indecomposable, for $j=1,2, \ldots, t$. If $M$ is indecomposable, then $t \leq 2$.

Proof. Let $0 \neq v \in M$ be such that $S=k v$. We consider now the canonical epimorphism $\pi: M \rightarrow M / S$. For each $j=1,2, \ldots, t$, we put $N_{j}=\pi^{-1} M_{j}$, the reciprocal image of $M_{j}$ under $\pi$. It is clear that $M=\sum_{j=1}^{t} N_{j}$ and $N_{i} \cap N_{j}=S$, if $i \neq j$. Moreover, $N_{j} / S=M_{j}$, for $j=1,2, \ldots, t$. It is clear that each $N_{j}$ is an indecomposable module.

Suppose now that $t \geq 3$. If we write $M=N_{1}+X$, where $X=\sum_{j=2}^{t} N_{j}$, it follows that $N_{1} \cap X=S=k v$ and, by the corollary above, there is a unique arrow $\sigma$, ending at $i_{S}$, such that $v \in \operatorname{Im} N_{1}(\sigma)$, and there is a unique arrow $\bar{\sigma}$, ending at $i_{S}$, with $\bar{\sigma} \neq \sigma$, such that $v \in \operatorname{Im} X(\bar{\sigma})$. So, applying lemma 4.4(i) to each $N_{j}$, for $j=2,3, \ldots, t$, and recalling that $\operatorname{Im} X(\bar{\sigma})=\sum_{j=2}^{t} \operatorname{Im} N_{j}(\bar{\sigma})$, we see that $v \in \operatorname{Im} N_{j}(\bar{\sigma})$, for all $j=2,3, \ldots, t$. On the other hand, if we write $X=N_{2}+\left(N_{3}+\ldots+N_{t}\right)$, then from theorem 4.5 it follows that $X=X_{1} \oplus X_{2}$, where $X_{i} \neq(0)$ for $i=1,2$, and $S \subset X_{1}$. Therefore, $M=N_{1}+\left(X_{1} \oplus X_{2}\right)=X_{2}+\left(N_{1}+X_{1}\right)$; since $M / S=$ $M_{1} \oplus M_{2} \oplus \ldots \oplus M_{t}$, and $N_{j}=\pi^{-1} M_{j}$, for $j=1,2, \ldots, t$, then $X_{2} \cap\left(N_{1}+X_{1}\right)=(0)$. This means that $M=X_{2} \oplus\left(N_{1}+X_{1}\right)$, which is a contradiction to the assumption that $M$ is indecomposable.

We observe that, given a module $M$ and a simple submodule $S$ of $M$, the results which we have gotten until now rely on the existence of paths in the quiver of $M$ ending at the vertex corresponding to $S$. By duality, we can get the dual results about the simple modules $T$ which are direct summands of top $M$.

\section{The multiplicity of the COMposition faCtors of SOME MOdUles}

We are now going to measure the multiplicity of a simple submodule $S$ of a given module $M$, when the quotient $M / S$ is of type $A_{n}$ for some $n \geq 2$, and also when $M / S$ is a direct sum of two submodules of type $A_{n}$, in case $\Lambda$ is a biserial algebra whose ordinary quiver has no loop and $\Lambda$-ind is determined by composition factors. Dually, we can measure the multiplicity of a simple module $T$ as a composition 
factor of $M$, where there is an epimorphism from $M$ to $T$ whose kernel is of type $A_{n}$ or is a direct sum of two submodules of type $A_{n}$.

We first need to introduce some notations and conventions which make reading easier. Let $Q^{\prime}$ be a quiver whose underlying graph is a diagram $A_{n}$, for $n \geq 3$. A vertex of $Q^{\prime}$ which belongs to exactly two arrows will be called an interior vertex of $Q^{\prime}$; and a vertex of $Q^{\prime}$ which belongs to exactly one arrow will be called an extreme vertex of $Q^{\prime}$. So, all vertices of $Q^{\prime}$, except two of them, are interior vertices. The extreme vertices can be distinguished in the following way: if $x$ is the origin of the unique arrow of $Q^{\prime}$ to which it belongs, we call $x$ the initial extreme; otherwise, we call it the terminal extreme. On the other hand, the interior vertices can be divided into three classes. If $x$ is an interior vertex such that it is the origin of the two arrows to which it belongs, we call $x$ a 2-source. If $x$ is the end of the two arrows to which it belongs, we call $x$ a 2 -sink. In the remaining case, $x$ is called a transit point.

We now fix the following convention: Let $M^{\prime}$ be a module of type $A_{n}$, with $n \geq 2$. If the quiver of $M^{\prime}$ contains a proper subquiver whose orientation is not important for the context, we shall represent this subquiver by points (vertices) and by edges.

Using the notations and conventions above, we have:

Lemma 5.1. Let $\Lambda=k\left(Q_{\Lambda}, R_{\Lambda}\right)$ be a representation-finite biserial algebra whose ordinary quiver $Q_{\Lambda}$ has no loop. Let $M$ be a $\Lambda$-module whose quiver is connected, and $S=k v \subset M$ be a simple submodule, where $0 \neq v \in M$. Suppose that $\bar{M}=M / S$ is of type $A_{n}, n \geq 2$, and that there is $\bar{v} \in \bar{M}\left(i_{S}\right)$ such that $\bar{S}=k \bar{v} \subset \bar{M}$. Then $M$ is one of the following $k$-representation of $\left(Q_{\Lambda}, R_{\Lambda}\right)$, relative to its support:

A.1
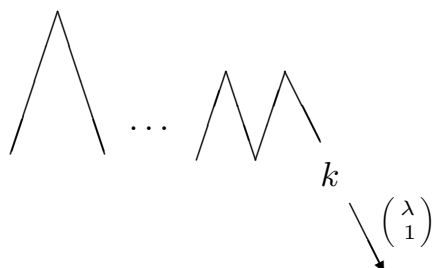

$k v \oplus k \bar{v}$
A.2

or
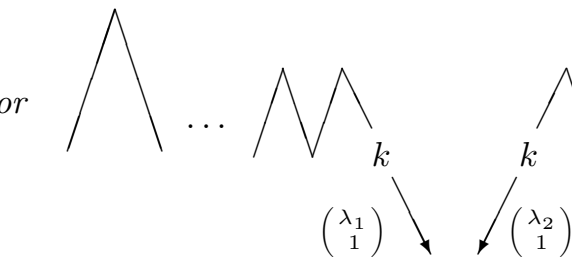

$k v \oplus k \bar{v}$

$(\lambda=0$ or $\lambda \cong \mathbb{1})$

$\left(\lambda_{i}=0, i=1,2\right.$, or $\left.\lambda_{i} \cong \mathbb{1}, i=1,2\right)$

Moreover, $S$ is a direct summand of $M$.

Proof. From the assumptions on $\bar{M}$, we write $M\left(i_{S}\right)=k v \oplus k \bar{v}$ and $M(x)=\bar{M}(x) \cong$ $k$, for all $x \in \operatorname{supp} M, x \neq i_{S}$. Since $S=k v \subset \operatorname{soc} M$ and $k \bar{v} \subset \bar{M}$, it follows that $M(\delta)(v)=\bar{M}(\delta)(v)=0$ for each arrow $\delta$, where $o(\delta)=i_{S}$, and $M(\alpha)=\bar{M}(\alpha)$, for each arrow $\alpha$ of $Q_{\Lambda}$, with $i_{S} \notin\{o(\alpha), e(\alpha)\}$. So, the quiver of $\bar{M}$ and, consequently, the quiver of $M$ does not contain arrows starting at $i_{S}$. Therefore, we conclude that $i_{S}$ is a sink in $Q_{M}$. Since the quiver $Q_{\bar{M}}$ is obtained from the quiver $Q_{M}$ by eventual exclusion of the arrows to which $i_{S}$ belongs, we need to analyse the neighborhood of $i_{S}$ in $Q_{\bar{M}}$.

$1 \stackrel{s t}{\text { ct }}$ case: $i_{S}$ is a 2-sink in $Q_{\bar{M}}$. Under this assumption, we infer that the quivers $Q_{M}$ and $Q_{\bar{M}}$ are equal and $i_{S}$ is a 2-sink of $Q_{M}$. Denote by $\sigma_{1}$ and $\sigma_{2}$, where $\sigma_{1} \neq \sigma_{2}$, arrows of $Q_{M}$ such that $e(\sigma)=i_{S}$ for $i=1,2$. Then, for $i=1,2, M\left(\sigma_{i}\right) \cong\left(\begin{array}{c}\lambda_{i} \\ 1\end{array}\right)$, 
where $\lambda_{i}=0$ or $\lambda_{i} \cong \mathbb{1}$. Suppose that $\lambda_{1}=0$ and $\lambda_{2} \cong \mathbb{1}$, and let $M_{1}$ and $M_{2}$ be the $k$-subrepresentations of $M$, which are represented, relative to its support, by
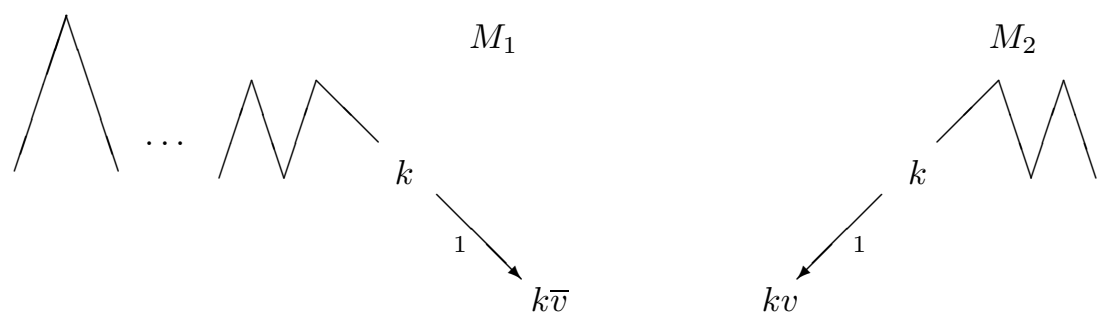

These $k$-representations are such that $k \bar{v} \subset \operatorname{soc} M_{1}$ and $k v \subset \operatorname{soc} M_{2}$. Consider $M_{1} \oplus M_{2}$, which is represented by

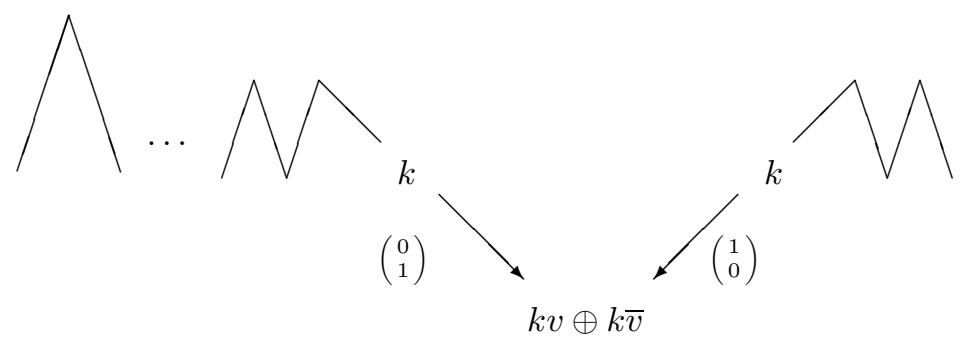

The family $f=\left(f_{x}\right)_{x}: M \longrightarrow M_{1} \oplus M_{2}$, given by $f_{x}=\mathbb{1}_{M(x)}$ if $x \neq i_{S}$ and $f_{i_{S}}(a v+b \bar{v})=a v+(b-a) \bar{v}$ for $a, b \in k$, defines an isomorphism of $k$-representations. Hence, $M / S \cong M_{1} \oplus M_{2} / k v$, and it is not of type $A_{n}$. Therefore, $\lambda_{i}=0$ for each $i=1,2$, or $\lambda_{i} \cong \mathbb{1}$ for each $i=1,2$, and $M$ is one of the representation in A.2. In the first case, it is very simple to verify that $S$ is a direct summand of $M$. In the second case, we have that the natural inclusion from $k v$ in $M$ is a monomorphism which admits as a left inverse morphism of $k$-representations the family $p=\left(p_{x}\right)_{x}: M \longrightarrow$ $S=k v$, defined by by $p_{x}=0$ if $x \neq i_{S}$ and $p_{i_{S}}(a v+b \bar{v})=(a-b) v$ for $a, b \in k$.

$2^{\text {nd }}$ case: $i_{S}$ is a terminal extreme in $Q_{\bar{M}}$. Let $\sigma: y \rightarrow i_{S}$ be the unique arrow of $Q_{\bar{M}}$ to which $i_{S}$ belongs. Then $M(\sigma)=\left(\begin{array}{c}\lambda \\ 1\end{array}\right)$, where $\lambda: k \rightarrow k v$ is zero or is a scalar multiple of the identity. Since $Q_{\Lambda}$ does not contain subquivers of type $\tilde{\mathrm{A}}_{m}$ without relations and verifies (SP)2, and since $\operatorname{supp} M=\operatorname{supp} \bar{M}$, it is easy to verify that $\sigma$ is the unique arrow of $Q_{\Lambda}$, ending at $i_{S}$, which is an arrow of $Q_{M}$. This implies that the quivers $Q_{M}$ and $Q_{\bar{M}}$ coincide, and we have case A.1. By the same arguments used in the first case, we obtain that the natural inclusion from $S=k v$ to $M$ splits, that is, $S$ is a direct summand of $M$.

In the following lemma, we consider a $k$-representation $M$ whose top admits a composition factor isomorphic to a direct summand of soc $M$.

Lemma 5.2. Let $\Lambda=k\left(Q_{\Lambda}, R_{\Lambda}\right)$ be a representation-finite biserial algebra whose ordinary quiver has no loop. Let $M$ be a $\Lambda$-module whose quiver $Q_{M}$ is connected. Consider $v \in M, v \neq 0$, and $S=k v \subset M$, a simple module. Suppose that $\bar{M}=M / S$ is of type $A_{n}, n \geq 2$. If there is $0 \neq \bar{v} \in \bar{M}\left(i_{S}\right)$ such that there exists an epimorphism 
from $\bar{M}$ to $\bar{S}=k \bar{v}$, then $M$ is one of the following $k$-representations, relative to its support:

B.1

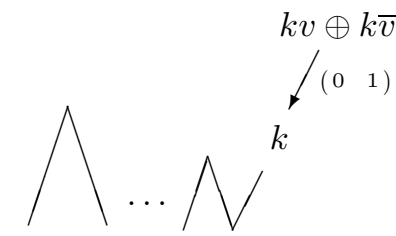

B.2

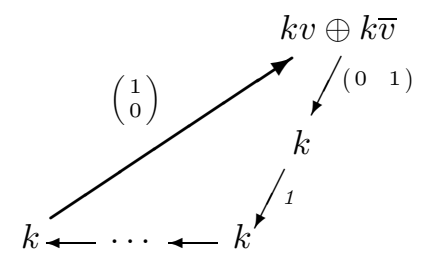

B.3

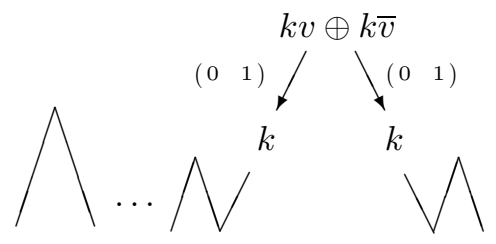

B.4

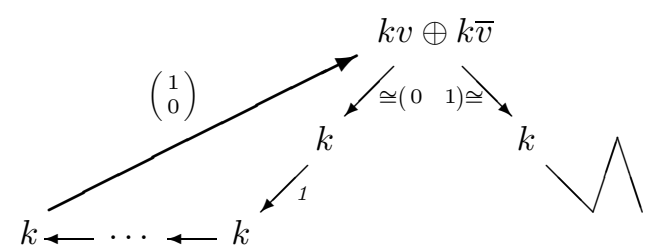

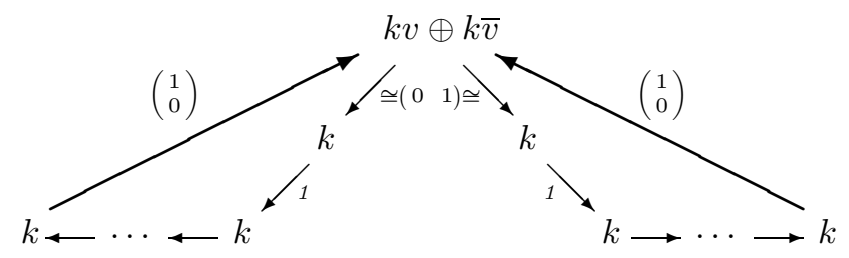

Furthermore, in cases B.1 and B.3, $S=k v$ is a direct summand of $M$.

Proof. As before, we can write $M\left(i_{S}\right)=k v \oplus k \bar{v}$ and $M(x)=\bar{M}(x) \cong k$, for all vertex $x \neq i_{S}$ of $Q_{M}$, and $M(\alpha)=\bar{M}(\alpha)$ for all arrows $\alpha$ with $i_{S} \notin\{o(\alpha), e(\alpha)\}$, and $M(\delta)(v)=0$ for each arrow $\delta$ with $o(\delta)=i_{S}$. From the hypothesis on $\bar{M}$ and $\bar{S}$, as in the previous lemma, it is easy to see that $i_{S}$ is a source of $Q_{\bar{M}}$. Moreover, from the epimorphism $M \rightarrow \bar{M} \rightarrow k \bar{v}$, it follows that $k \bar{v} \cap \sum_{\gamma} \operatorname{Im} M(\gamma)=(0)$, for each arrow $\gamma$, where $e(\gamma)=i_{S}$. We have now some cases, according to which kind of source $i_{S}$ is of $Q_{\bar{M}}$ and if there is an arrow ending at $i_{S}$ in $Q_{M}$.

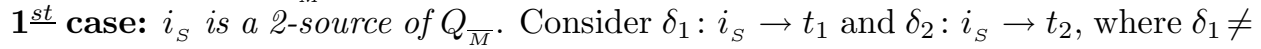
$\delta_{2}$ (hence, $\left.t_{1} \notin\left\{i_{S}, t_{2}\right\}\right)$, such that $\bar{M}\left(\delta_{1}\right) \cong \mathbb{1} \cong \bar{M}\left(\delta_{2}\right)$. So, $M\left(\delta_{1}\right) \cong\left(\begin{array}{ll}0 & 1\end{array}\right) \cong$ $M\left(\delta_{2}\right)$. According to whether $i_{S}$ is an end point of arrows in $Q_{M}$, we have the following subcases.

1.1: There is no arrow in $Q_{M}$ ending at $i_{S}$ (i.e, $i_{S}$ is a 2-source of $Q_{M}$ ). This condition implies that the quivers $Q_{M}$ and $Q_{\bar{M}}$ coincide, and so $M$ is the $k$-representation in B.3.

1.2: There is in $Q_{M}$ exactly one arrow $\gamma$ with $e(\gamma)=i_{S}$. Since supp $M=\operatorname{supp} \bar{M}$, $\bar{M}$ is of type of $A_{n}$ and the condition (SP) 2 is satisfied, it follows that $o(\gamma)=x \neq i_{S}$ is an extreme vertex of $Q_{\bar{M}}$. Hence, since $Q_{\Lambda}$ does not contain loops, double arrows or 
subquivers of type $\tilde{\mathrm{A}}_{r}$ without relations (since $\Lambda$ is representation-finite), it results that there is, in $Q_{\bar{M}}$, a path from $i_{S}$ to $x$ (this path is unique and $x$ is its terminal extreme). Then there exists an oriented cycle in $Q_{M}$ containing this path and $\gamma$. On the other hand, since $\gamma: x \rightarrow i_{S}$ is exactly the one arrow of $Q_{\Lambda}$ such that $M(\gamma) \neq 0$, it follows that $k \bar{v} \cap \operatorname{Im} M(\gamma)=k \bar{v} \cap \sum_{\gamma} \operatorname{Im} M(\gamma)=(0)$, and so, by a convenient choice, we have that $M(\gamma) \cong\left(\begin{array}{l}1 \\ 0\end{array}\right)$, that is, $M$ is the $k$-representation in B.4.

1.3: There are, in $Q_{M}$, two different arrows $\gamma_{1}$ and $\gamma_{2}$ with $e\left(\gamma_{i}\right)=i_{S}$, for $i=1,2$. Let $x_{i}=o\left(\gamma_{i}\right)$, for $i=1,2$. Then, $x_{1} \neq x_{2}$ and $i_{S} \notin\left\{x_{1}, x_{2}\right\}$. As in 1.2, for each $i=1,2, x_{i}$ is an extreme vertex of $Q_{\bar{M}}$ and, hence, each $x_{i}$ is the terminal vertex of the unique path in $Q_{\bar{M}}$ joining $i_{S}$ to $x_{i}$. So, this means that $Q_{\bar{M}}$ is the union of these paths and, consequently, $Q_{M}$ is the union of the two oriented cycles which are determined by the mentioned paths above and the arrows $\gamma_{i}$, for $i=1,2$, having a unique common vertex, the vertex $i_{S}$. As in case 1.2 , by a convenient choice, we have that $M\left(\gamma_{i}\right)=\left(\begin{array}{l}1 \\ 0\end{array}\right)$ for $i=1,2$, and, hence, $M$ is the $k$-representation in B.5. $2^{\frac{n d}{d}}$ case: $i_{S}$ is an initial extreme of $Q_{\bar{M}}$. Let $\delta: i_{S} \rightarrow t$ be the unique arrow such that $\bar{M}(\delta) \cong \mathbb{1}$. It is clear that $\delta$ is the unique arrow of $Q_{M}$ starting at $i_{S}$, and so, $M(\delta) \cong\left(\begin{array}{ll}0 & 1\end{array}\right)$. By the analysis of whether the $Q_{M}$ contains arrows ending at $i_{S}$, it is easy to see, using analogous arguments, that either $Q_{M}$ and $Q_{\bar{M}}$ coincide (and, in this case, $M$ is the $k$-representation in B.1), or $Q_{\bar{M}}$ is a proper subpath of $Q_{M}$, obtained by elimination of one arrow ending at $i_{S}$. In this last case the quiver of $M$ is an oriented cycle and hence $M$ is the representation in B.2.

In both cases B.1 and B.3, it is very simple to verify that the natural inclusion of $S=k v$ in $M$ is a splittable monomorphism.

Lemma 5.3. Let $\Lambda=k\left(Q_{\Lambda}, R_{\Lambda}\right)$ be a biserial representation-finite type algebra whose ordinary quiver $Q_{\Lambda}$ has no loop. Let $M$ be a $k$-representation whose quiver is connected. Consider $v \in M$ and the simple submodule of $M, S=k v$. Suppose that $\bar{M}=M / S$ is of type $A_{n}, n \geq 2$. If there is $0 \neq \bar{v} \in \bar{M}\left(i_{S}\right)$ such that the simple module $\bar{S}=k \bar{v}$ is not a direct summand of the module soc $\bar{M} \oplus$ top $\bar{M}$, then $M$ is one of the following $k$-representations:

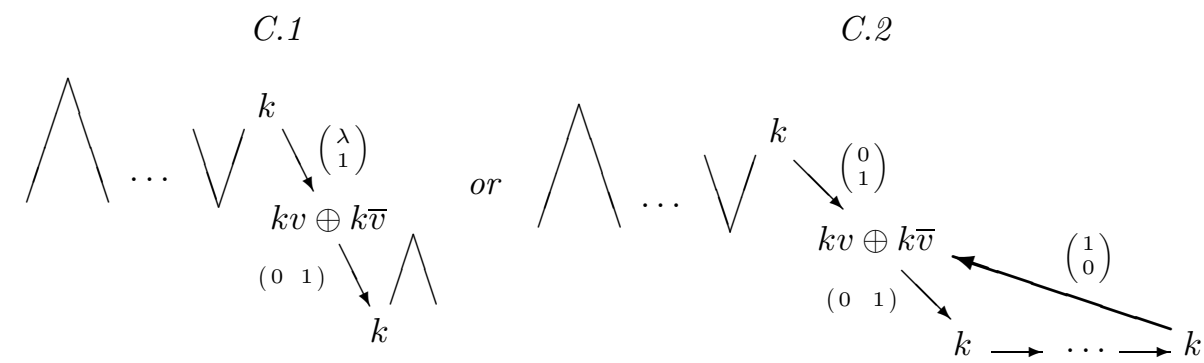

$$
(\lambda=0 \text { or } \lambda \cong \mathbb{1})
$$

In case C.1, $S=k v$ is a direct summand of $M$.

Proof. The proof of this lemma is very similar to the proof of the previous lemmas, and we leave it for the reader.

Proposition 5.4. Let $\Lambda=k\left(Q_{\Lambda}, R_{\Lambda}\right)$ be a representation-finite biserial $k$-algebra such that the ordinary quiver $Q_{\Lambda}$ does not have any loop. Let $M$ be an indecomposable $\Lambda$-module and $S$ be a simple submodule of $M$ such that $\bar{M}=M / S$ is of type $A_{n}$, 
with $n \geq 3$. If $\bar{M}\left(i_{S}\right)$ is non-zero, then $M$ is one of the $k$-representations in B.2, B.4, B.5 in lemma 5.2 or C.2 in lemma 5.3.

Proof. Let $0 \neq v \in M$ be such that $S=k v$ and $0 \neq \bar{v} \in \bar{M}\left(i_{S}\right)$. Consider the simple module $k \bar{v}$. Since $k \bar{v}$ is a composition factor of $\bar{M}$ and $Q_{M}$ is connected (since $M$ is indecomposable), by lemma $5.1, k \bar{v}$ is a composition factor of top $M$ or is not a composition factor of $\operatorname{soc} \bar{M} \oplus \operatorname{top} M$. If $k \bar{v}$ is a composition factor of top $M$, from lemma 5.2 it follows that $M$ is one of the representations in B.2, B.4 or B.5. If, instead, $k \bar{v}$ is not a composition factor of the module $\operatorname{soc} \bar{M} \oplus \operatorname{top} M$, then it is not a composition factor of the module $\operatorname{soc} \bar{M} \oplus \operatorname{top} \bar{M}$, and from lemma 5.3, it follows that $M$ is the representation in C.2.

Proposition 5.5. Let $\Lambda$ be a biserial algebra whose ordinary quiver has no loop. Suppose that the category $\Lambda$-ind is determined by composition factors. Let $M$ be an indecomposable $\Lambda$-module, where $l(M) \geq 3$, and $S$ be a simple $\Lambda$-module such that $S \subset M$ and $\bar{M}=M / S$ is of type $A_{n}$. Then $M$ is multiplicity-free.

Proof. Since $\Lambda$-ind is determined by composition factors, then by remark $1.1 \Lambda$ is a representation-finite biserial algebra and, therefore, $\Lambda \cong k\left(Q_{\Lambda}, R_{\Lambda}\right)$, under the conditions of the proposition and the conditions of remarks 4.1 and 4.2 . Under the assumption on $M$ and $\bar{M}$, we have that $M(x) \cong \bar{M}(x) \cong k$, for all $x \in \operatorname{supp} M$, $x \neq i_{S}$. To get the result we need to prove that $\bar{M}\left(i_{S}\right)=0$. Suppose that $\bar{M}\left(i_{S}\right) \cong k$. By proposition 5.4, $M$ is one of the $k$-representations in B.2, B.4, B.5 or C.2. In any of these cases, it is immediate to see that there is a subrepresentation $N_{1}$ of $\bar{M} \cong M / k v$ and a subrepresentation $N_{2}$ of $M$, where $l\left(N_{i}\right) \geq 2$, for each $i=1,2$, which are represented, relative to its support, by

$N_{1}$

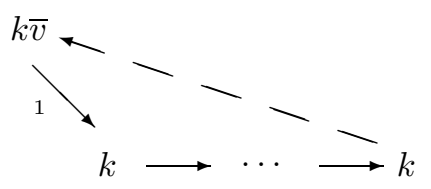

$N_{2}$

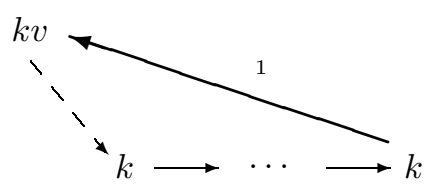

where the dotted arrows indicate that such arrows do not belong to the quiver of $N_{i}$, for each $i=1,2$ (that is, the $k$-morphisms to which they correspond are zero). The $k$-representations $N_{1}$ and $N_{2}$ are of type $A_{r}$, where $r=l\left(N_{1}\right)=l\left(N_{2}\right)$, and so, they are indecomposable modules having the same composition factors, but they are not isomorphic ( since $\operatorname{soc} N_{2} \cong k v \neq \operatorname{soc} N_{1}$ ). We obtain a contradiction to the assumption that $\Lambda$-ind is determined by composition factors. Therefore, $M(x) \cong k$, for all vertices of the quiver of $M$, that is, $M$ is multiplicity free.

Proposition 5.6. Let $\Lambda$ be a biserial k-algebra whose ordinary quiver does not have any loop and such that $\Lambda$-ind is determined by composition factors. Let $M$ be an indecomposable $\Lambda$-module, $l(M) \geq 3$, and $S$ be a simple submodule of $M$ such that $\bar{M}=M / S=N_{1} \oplus N_{2}$, where the $N_{i}$ are of type $A_{n_{i}}, n_{i} \geq 1$, for $i=1,2$. Then $S$ is a composition factor of $M$ with multiplicity one.

Proof. As in the proof of proposition $5.5, \Lambda \cong k\left(Q_{\Lambda}, R_{\Lambda}\right)$ satisfies the same conditions mentioned there. Let $0 \neq v \in M$ be such that $S=k v$. We consider the canonical 
epimorphism $\pi: M \rightarrow \bar{M}$ and we denote by $M_{j}^{\prime}$, for $j=1,2$, the reciprocal image of $N_{j}$ by $\pi$. Then, $M=M_{1}^{\prime}+M_{2}^{\prime}$ and $M_{1}^{\prime} \cap M_{2}^{\prime}=k v$. Moreover, for each $j=1,2$, $M_{j}^{\prime}(x)=N_{j}(x) \cong k$, where $x \in \operatorname{supp} N_{j}, x \neq i_{S}$, and $M\left(i_{S}\right)=k v \oplus N_{1}\left(i_{S}\right) \oplus N_{2}\left(i_{S}\right)$.

Suppose, for $j=1$, that $N_{1}\left(i_{S}\right) \cong k$. Then, $M_{1}^{\prime}\left(i_{S}\right)=k v \oplus N_{1}\left(i_{S}\right) \cong k v \oplus k$. On the other hand, since $M$ is an indecomposable module, by corollary 4.6 , there is a unique arrow $\sigma: y \rightarrow i_{S}$ such that $v \in \operatorname{Im} M_{1}^{\prime}(\sigma)$; then $l\left(M_{1}\right) \geq 3$ and the quiver of $M_{1}^{\prime}$ is connected. Since $M_{1}^{\prime} / S=N_{1}$ is of type $A_{n_{1}}$, it follows that $M_{1}^{\prime}$ is one of the representations in lemma $5.1,5.2$ or 5.3 . But, since $\Lambda$-ind is determined by composition factors, from the proof of proposition 5.5, we infer that $M_{1}^{\prime}$ is one of the representations in A.1, A.2, B.1 or B.3. In any of these cases, $S$ is a direct summand of $M_{1}^{\prime}$ and, consequently, a direct summand of $M$, which contradicts the indecomposability of $M$. So, $M\left(i_{S}\right)=k v$, that is, $S$ has multiplicity one as a composition factor of $M$.

\section{THE MAIN THEOREMS}

The methods developed in the previous sections allow us now to prove the following results.

Theorem 6.1. Let $\Lambda=k\left(Q_{\Lambda}, R_{\Lambda}\right)$ be a representation-finite biserial algebra. Suppose that the ordinary quiver of $\Lambda$ does not have any loop and that all oriented cycles of $Q_{\Lambda}$ are in $R_{\Lambda}$. If $M$ is a multiplicity-free indecomposable $\Lambda$-module, then $M$ either is a projective-injective non-uniserial module or is of type $A_{n}$, where $n=l(M)$.

Proof. We first observe that $\left(Q_{\Lambda}, R_{\Lambda}\right)$ was chosen satisfying the conditions (SP) of theorem 4.1, and also the conditions of remark 1.1. Since $M$ is a multiplicity-free indecomposable module, then $M(x) \cong k$, for each $x \in \operatorname{supp} M$. We shall proceed by induction on the length of the multiplicity-free indecomposable modules. It is clear that each indecomposable module $M$, where $l(M)=n \leq 3$, is of type $A_{n}$, since it is a local or colocal module.

Suppose now that $M$ is an indecomposable multiplicity-free module, with $l(M)=$ $n \geq 4$, and that the result is true for all indecomposable multiplicity-free module $N$ such that $1 \leq l(N)<l(M)$. Let $S$ be a simple submodule of $M$ and consider the quotient $M / S$. If $M / S$ is indecomposable then, by the inductive assumption, $M / S$ is of type $A_{n-1}$. Let $\left\{i_{1}, i_{2}, \ldots, i_{n-1}\right\}=\operatorname{supp} M / S$ be ordered in such a way that $i_{1}$ and $i_{n-1}$ are the extreme vertices of the quiver of $M / S$. Since $S \subset M$ and $M$ is an indecomposable multipliciy-free module (therefore, $Q_{M}$ is connected), then there is at least one arrow $\sigma: i_{j} \rightarrow i_{S}$, for some $j, 1 \leq j \leq n-1$ (and at most two), such that $\sigma$ is in $Q_{M}$. From the conditions on $\left(Q_{\Lambda}, R_{\Lambda}\right)$, it follows that $o(\sigma) \in\left\{i_{1}, i_{n-1}\right\}$.

Suppose that the quiver of $M / S$ is a path, for example a path from $i_{n-1}$ to $i_{1}$. Since $Q_{\Lambda}$ contains no subquiver of type $\tilde{\mathrm{A}}_{r}$ without relations or loops, then $Q_{M}$ does not contain another arrow $\bar{\sigma} \neq \sigma$ of $Q_{\Lambda}$ ending at $i_{S}$. So, the underlying diagram of $Q_{M}$ is of type $A_{n}$, and $M$ is of type $A_{n}$, where $n=l(M)$. Otherwise, if the quiver of $M / S$ is of type $A_{n-1}$, but is not a path, then there is at least one vertex $i_{m}$, $2 \leq m \leq n-2$, such that $i_{m}$ is either a 2 -source or a 2 -sink of $Q_{M / S}$.

Let us assume first that $i_{m}$ is the unique 2-source of $Q_{M / S}$. Then $Q_{M / S}$ is the union of a path $\mu$ from $i_{m}$ to $i_{1}$ and a path $\nu$ from $i_{m}$ to $i_{n-1}$ in $Q_{M / S}$. So, we infer that either $\sigma$ is the unique arrow of $Q_{\Lambda}$ ending at $i_{S}$ which is an arrow of $Q_{M}$ or, besides $\sigma, Q_{M}$ contains another arrow $\bar{\sigma}$, where $e(\bar{\sigma})=i_{S}, o(\sigma)=i_{1}$ and $o(\bar{\sigma})=i_{n-1}$ such that $\sigma \mu-\bar{\sigma} \nu \in R_{\Lambda}$ (by (SP)4). In the first alternative, it follows imediately 
that the underlying graph of $Q_{M}$ is a diagram $A_{n}$, and so, $M$ is of type $A_{n}$. The second alternative implies that $Q_{M}$ is

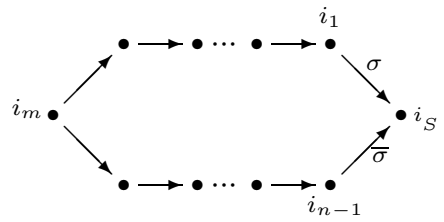

and, consequently, $M$ is a projective-injective non-uniserial module.

Otherwise, if $i_{m}$ is not the unique 2-source or $i_{m}$ is a 2 -sink of $Q_{M / S}$, then $\sigma$ is the unique arrow of $Q_{\Lambda}$ ending at $i_{S}$ which is an arrow of $Q_{M}$, since $Q_{\Lambda}$ contains no subquiver of type $\tilde{\mathrm{A}}_{r}$ without relations. This implies that $M$ is of type $A_{n}$.

Finally, we need to consider the case in which $M / S$ is a decomposable module. By theorem 4.7, $M / S=M_{1} \oplus M_{2}$, where, for each $i=1,2, M_{i}$ is an indecomposable non-projective module. Moreover, each $M_{i}$ is multiplicity-free and they have disjoint supports. Denoting by $n_{i}$ the length of $M_{i}, i=1,2$, we have that $M_{i}$ is of type $A_{n_{i}}$ (by the induction assumption, because $\left.l\left(M_{i}\right)<l(M), i=1,2\right)$. If we now consider the canonical epimorphism $\pi: M \rightarrow M / S$, we can write $M=N_{1}+N_{2}$, where $N_{1} \cap N_{2}=S$ and $N_{i}$ is the reciprocal image of $M_{i}$ under $\pi$. Then, since $M\left(i_{S}\right) \cong k$ and supp $N_{1} \cap \operatorname{supp} N_{2}=\left\{i_{S}\right\}$, by corollary 4.6, there is a unique arrow $\sigma_{1}: y_{1} \rightarrow i_{S}$ such that $N_{1}\left(\sigma_{1}\right) \cong \mathbb{1}$ and there is a unique arrow $\sigma_{2}: y_{2} \rightarrow i_{S}$, where $\sigma_{2} \neq \sigma_{1}$ and $y_{1} \notin\left\{y_{2}, i_{s}\right\}$, such that $N_{2}\left(\sigma_{2}\right) \cong \mathbb{1}$. Moreover, for each $i=1,2, y_{i} \in \operatorname{supp} M_{i}$ is a extreme vertex of the quiver $Q_{M_{i}}$. So, we conclude that $Q_{M}$ has for its underlying graph a diagram $A_{n}$, and the theorem is proved.

Theorem 6.2. Let $\Lambda$ be a connected, basic biserial $k$-algebra whose ordinary quiver does not have a loop. Suppose that $\Lambda$-ind is determined by composition factors. Then each indecomposable $\Lambda$-module is multiplicity-free.

Proof. Since $\Lambda$-ind is determined by composition factors, by remark $1.1, \Lambda$ is a representation-finite biserial algebra, and so, we choose $\Lambda \cong k\left(Q_{\Lambda}, R_{\Lambda}\right)$, where $\left(Q_{\Lambda}, R_{\Lambda}\right)$ satisfies the conditions (SP) of theorem 4.1 and those of proposition 3.1. Moreover, according to remark 4.2, all oriented cycles of $k Q_{\Lambda}$ are in $R_{\Lambda}$. We shall proceed by induction on the length of the indecomposable modules. Let $M$ be in $\Lambda$-ind and such that $l(M) \leq 3$. Is easy to verify that each indecomposable of length less than or equal to 3 is a local or colocal module, and, by theorem 4.3 , it follows that $M$ is multiplicity-free. Suppose that $M$ is indecomposable such that $l(M) \geq 4$ and that the result is true for each indecomposable module $N$ such that $1 \leq l(N)<l(M)$. By duality it is enough to prove that if $S$ is a composition factor of $M$ which is a direct summand of $\operatorname{soc} M$ or $S$ is not a composition factor of soc $M \oplus$ top $M$, then the multiplicity of $S$ in $M$ is one.

Let $S$ be a simple submodule of $M$ and consider the quotient $M / S$. If $M / S$ is indecomposable, by the induction assumption it follows that $M / S$ is multiplicityfree. Since $M / S$ is not a projective module (since $M$ is indecomposable), then, by theorem 6.1, it is of type $A_{n-1}$, where $n=l(M)$, and so, by proposition 5.5 , the multiciplity of $S$ in $M$ is one. If $M / S$ is not indecomposable, then, by theorem 4.7, it follows that $M / S=M_{1} \oplus M_{2}$, where each $M_{i} \neq(0)$ is an indecomposable nonprojective module, for $i=1,2$. Again by the induction assumption and theorem 6.1, each $M_{i}$ is of type $A_{n_{i}}$, where $n_{i}=l\left(M_{i}\right)$. Hence, by proposition 5.6 , the multiplicity 
of $S$ in $M$ is one. So, we conclude that each simple submodule of $M$ has multiplicity one as a composition factor of $M$.

Let $T$ be a simple module which is a composition factor of $M$ but not a composition factor of the module soc $M \oplus \operatorname{top} M$. Suppose that the multiplicity of $T$ in $M$ is greater than or equal to 2 , that is, $\operatorname{dim}_{k} M\left(i_{T}\right) \geq 2$. Let $S$ be a simple submodule of $M$. By the proof above we have that $\operatorname{dim}_{k} M\left(i_{S}\right)=1$, and so $S \neq T$. Consider the quotient $M / S$. Since $\operatorname{dim}_{k}(M / S)\left(i_{T}\right) \geq 2$, by the induction assumption (for $l(M / S)<l(M))$ and by theorem 4.7, it follows that $M / S=M_{1} \oplus M_{2}$, where $M_{i} \neq(0)$, $i=1,2$, are indecomposable non-projective modules, whose $\operatorname{dim}_{k} M_{i}\left(i_{T}\right)=1$, for each $i=1,2$, and $\operatorname{dim}_{k} M\left(i_{T}\right)=2$. Moreover, since $l\left(M_{i}\right)<l(M)$, for each $i=1,2$, by the induction assumption and theorem 6.1 , each $M_{i}$ is of type $A_{n_{i}}$, where $n_{i}=l\left(M_{i}\right) \geq 1$. Now let $\pi: M \rightarrow M / S$ be the canonical epimorphism, and let the submodules $N_{i}=\pi^{-1} M_{i}, i=1,2$, be such that $M=N_{1}+N_{2}, N_{1} \cap N_{2}=S=k v$, where $v \in M\left(i_{S}\right)$, and $N_{i} / S=M_{i}$, for $i=1,2$. From corollary 4.6, it follows that there are arrows $\sigma_{1}: y_{1} \rightarrow i_{S}$ and $\sigma_{2}: y_{2} \rightarrow i_{S}$, where $\sigma_{1} \neq \sigma_{2}$ (hence, $y_{1} \neq i_{S}$ and $\left.y_{2} \notin\left\{y_{1}, i_{S}\right\}\right)$, such that $\operatorname{Im} N_{i}\left(\sigma_{i}\right)=k v$, for each $i=1,2$ and $N_{i}\left(\sigma_{j}\right)=0$ if $i \neq j$. Then, each $N_{i}$ is of type $A_{n_{i}+1}$ and $i_{S}$ is a terminal extreme of the quiver $Q_{N_{i}}$, for $i=1,2$. Hence, $i_{S}$ is a 2 -sink of $Q_{M}$ and $\left\{i_{S}, i_{T}\right\} \subset \operatorname{supp} N_{1} \cap \operatorname{supp} N_{2}$. We claim that if $z \in \operatorname{supp} N_{1} \cap \operatorname{supp} N_{2}, z \neq i_{S}$, then $z$ is a transit point of $Q_{N_{1}}$ and of $Q_{N_{2}}$. In fact, since $\operatorname{soc} N_{i} \subset \operatorname{soc} M$, for each $i=1,2$, and each composition factor of $\operatorname{soc} M$ has multiplicity one in $M$, then the simple module $S_{z}$ is not a composition factor of $\operatorname{soc} N_{i}$, for all $i=1,2$. So, $z$ is not a sink of $Q_{N_{1}}$, nor of $Q_{N_{2}}$. On the other hand, since top $M=$ top $N_{1} \oplus$ top $N_{2}$ and each composition factor of top $M$ has multiplicity one in $M$ (for duality of the $\operatorname{soc} M$ ), then, for each $i=1,2$, no direct summand of top $N_{i}$ is isomorphic to $S_{z}$. Therefore, $z$ is not a source of $Q_{N_{1}}$, nor of $Q_{N_{2}}$. Our claim is proved.

Therefore, the set supp $N_{1} \cap$ supp $N_{2}$ contains the vertices of $M$ which are transit point of $Q_{N_{1}}$ and of $Q_{N_{2}}$. So, the neighbourhood of these vertices $z \neq i_{S}$ in each of these subquivers has the following figure: $x_{i} \stackrel{\alpha_{i}}{\rightarrow} z \stackrel{\beta_{i}}{\rightarrow} w_{i}$ such that $\beta_{i} \alpha_{i} \notin R_{\Lambda}\left(x_{i}, w_{i}\right)$, for each $i=1,2$. From the condition (SP)2, it follows that if $\alpha_{1}=\alpha_{2}$ then $\beta_{1}=\beta_{2}$, and if $\alpha_{1} \neq \alpha_{2}$, then $\beta_{1} \neq \beta_{2}, \beta_{2} \alpha_{1} \in R_{\Lambda}\left(x_{1}, w_{2}\right)$ and $\beta_{1} \alpha_{2} \in R_{\Lambda}\left(x_{2}, w_{1}\right)$. Thus the neighbourhood of each $z \in \operatorname{supp} N_{1} \cap \operatorname{supp} N_{2}$, with $z \neq i_{S}$, is one of the following figures:

(a) $\underset{\dot{x}_{1}}{\stackrel{\alpha_{1}}{\rightarrow}} \stackrel{\stackrel{\beta_{1}}{\rightarrow}}{\dot{w}_{1}}$ with $\beta_{1} \alpha_{1} \notin R_{\Lambda}$ or (b)

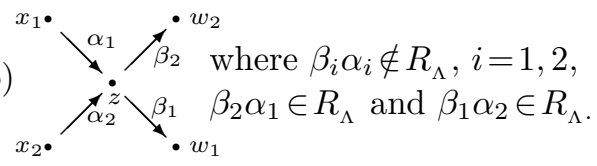

We observe that in the case (a) we have that $\left\{x_{1}, w_{1}\right\} \subset \operatorname{supp} N_{1} \cap \operatorname{supp} N_{2}$. For each $z \in \operatorname{supp} N_{1} \cap \operatorname{supp} N_{2}$, where $z \neq i_{S}$, and for each $i=1,2$, let $A_{i}\left(i_{S}, z\right)$ be the walk of minimal length of the quiver $N_{i}$ joining $i_{S}$ to $z$. Then, for each $i$, $A_{i}\left(i_{S}, z\right)$ contains the arrow $\sigma_{i}$ and is, in particular, a subquiver of type $A_{r_{i}}$, where $r_{i}=l\left(A_{i}\left(i_{S}, z\right)\right)+1$ and $l\left(A_{i}\left(i_{S}, z\right)\right)$ denotes the number of arrows of $A_{i}\left(i_{S}, z\right)$. Now, we choose, for each $i=1,2, z_{i} \in \operatorname{supp} N_{1} \cap \operatorname{supp} N_{2}$, where $z_{i} \neq i_{S}$, such that $l\left(A_{i}\left(i_{S}, z_{i}\right)\right)$ is the least among the vertices $z \neq i_{S}$ which are in supp $N_{1} \cap \operatorname{supp} N_{2}$. This choice implies that we need to annalyse two cases: $z_{1}=z_{2}$ or $z_{1} \neq z_{2}$.

$1 \stackrel{s t}{\text { case: }} z_{1}=z_{2}=z_{0}$. The minimality of the length of $A_{i}\left(i_{S}, z_{0}\right), i=1,2$, implies that the neighborhood of $z_{0}$ in $Q_{M}$ is as in figure (b) and that $i_{S}$ and 
$z_{0}$ are the unique common vertices of $A_{1}\left(i_{S}, z_{0}\right)$ and $A_{2}\left(i_{S}, z_{0}\right)$. Then $Q_{M}$ and, consequently, $Q_{\Lambda}$, contain one of the following subquivers, which are the union of the walks $A_{i}\left(i_{S}, z_{0}\right), i=1,2$ :

(c)

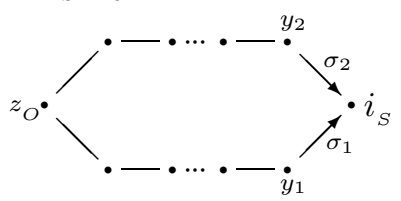

(d)

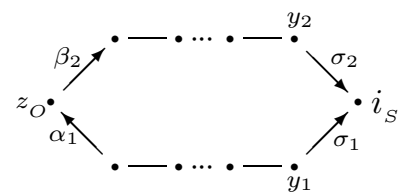

where in (c) $z_{0}$ is a 2 -source or a 2 -sink.

In (c), since $Q_{\Lambda}$ contains no subquivers of type $\tilde{\mathrm{A}}_{r}$ without relations, it is easy to see that each $A_{i}\left(i_{S}, z_{0}\right), i=1,2$, is a path from $z_{0}$ to $i_{S}$. So, they are parallel and such that $A_{1}\left(i_{S}, z_{0}\right)-A_{2}\left(i_{S}, z_{0}\right) \notin R_{\Lambda}$ (for $\left.A_{i}\left(i_{S}, z_{0}\right) \alpha_{i} \notin R_{\Lambda}\right)$, which is a contradiction to condition (SP) 4 of theorem 4.1. If we have case (d), we construct the families $N^{\prime}=\left(N^{\prime}(x)_{x}, N^{\prime}(\delta)_{\delta}\right)$ and $N^{\prime \prime}=\left(N^{\prime \prime}(x)_{x}, N^{\prime \prime}(\delta)_{\delta}\right)$, where $x$ denotes the vertices of $Q_{\Lambda}$ and $\delta$ their arrows, defined by:

$$
\begin{gathered}
N^{\prime}(x)= \begin{cases}N_{2}(x) & \text { if } x \text { is in } A_{2}\left(i_{S}, z_{0}\right), x \neq i_{S}, \\
N_{1}(x) & \text { if } x \text { is in } A_{1}\left(i_{S}, z_{0}\right), x \notin\left\{z_{0}, i_{S}\right\}, \\
k v & \text { if } x=i_{S}, \\
(0) & \text { for the other vertices, }\end{cases} \\
N^{\prime}(\delta)= \begin{cases}N_{2}(\delta) & \text { if } \delta \text { is in } A_{2}\left(i_{S}, z_{0}\right), \\
N_{1}(\delta) & \text { if } \delta \text { is in } A_{1}\left(i_{S}, z_{0}\right), \delta \neq \alpha_{1}, \\
0 & \text { for the other arrows, }\end{cases} \\
N^{\prime \prime}(x)= \begin{cases}N_{2}(x) & \text { if } x \text { is in } A_{2}\left(i_{S}, z_{0}\right), x \notin\left\{z_{0}, i_{S}\right\}, \\
N_{1}(x) & \text { if } x \text { is in } A_{1}\left(i_{S}, z_{0}\right), x \neq i_{S}, \\
k v & \text { if } x=i_{S}, \\
(0) & \text { for the other vertices, }\end{cases} \\
N^{\prime \prime}(\delta)= \begin{cases}N_{2}(\delta) & \text { if } \delta \text { is in } A_{2}\left(i_{S}, z_{0}\right), \delta \neq \beta_{2}, \\
N_{1}(\delta) & \text { if } \delta \text { is in } A_{1}\left(i_{S}, z_{0}\right), \\
0 & \text { for the other arrows. }\end{cases}
\end{gathered}
$$

By the definition of $N^{\prime}$ and $N^{\prime \prime}$, it is easy to verify that they are $k$-representations such that $\operatorname{supp} N^{\prime}=\operatorname{supp} N^{\prime \prime}$. Moreover, the quivers $Q_{N^{\prime}}$ and $Q_{N^{\prime \prime}}$ are obtained from the quiver in (d) by deleting, respectively, the arrows $\alpha_{1}$ and $\beta_{2}$; and it follows that $N^{\prime}\left(N^{\prime \prime}\right)$ is a module of type $A_{r}$, where $r=l\left(A_{1}\left(i_{S}, z_{0}\right)\right)+l\left(A_{2}\left(i_{S}, z_{0}\right)\right)$, whose top (respectively, whose soc) contains a direct summand isomorphic to the simple module $S_{z_{0}}$, since the vertex $z_{0}$ is a initial extreme of $Q_{N^{\prime}}$ (respectively, since $z_{0}$ is a terminal extreme of $\left.Q_{N^{\prime \prime}}\right)$. So we construct two multiplicity-free modules $\left(N^{\prime}\right.$ and $\left.N^{\prime \prime}\right)$ having the same composition factors, but non-isomorphic, which is a contradiction.

$2^{\frac{n d}{d}}$ case: $z_{1} \neq z_{2}$. From the minimality of the length of $A_{1}\left(i_{S}, z_{1}\right)$ and of $A_{2}\left(i_{S}, z_{2}\right)$, it follows that $i_{S}$ is the unique common vertex of them and, analogously to the first case, the neighbourhood of $z_{1}$ and of $z_{2}$ in $Q_{M}$ are as in figure (b). So, we indicate their arrows as well as the vertices of the neighbourhood of each $z_{i}$, using double indices, in the following way: the first index $i$ corresponds to the vertex $z_{i}$ and the second one $j$ to which $Q_{N_{j}}$ belongs, as the following figure shows: 


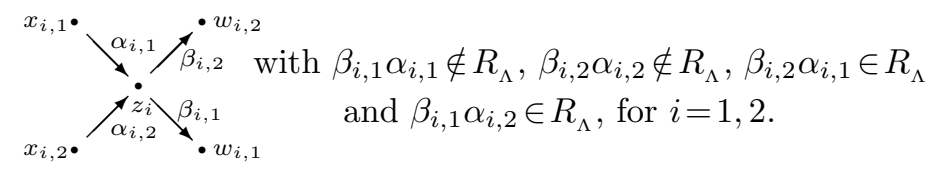

Since, for each $i=1,2, Q_{N_{i}}$ is connected, we consider the walk $A_{1}\left(z_{1}, z_{2}\right)$ in $Q_{N_{1}}$ and the walk $A_{2}\left(z_{1}, z_{2}\right)$ in $Q_{N_{2}}$, such that they are the walks of the least length, in each corresponding quiver, joining $z_{1}$ to $z_{2}$. It is clear, by the assumption on minimality of the length, that $z_{1}$ and $z_{2}$ are their unique common vertices. So, we have many different possibilities, according to whether the arrows $\alpha_{i, j}$ and $\beta_{i, j}$, for $i, j=1,2$, belong (or not) to the walks $A_{i}\left(i_{S}, z_{i}\right)$ and $A_{i}\left(z_{1}, z_{2}\right)$, which are the following.

2.1: For all $i=1,2, A_{i}\left(i_{S}, z_{i}\right)$ contains the arrow $\beta_{i, i}$. Under this condition, each walk $A_{i}\left(z_{1}, z_{2}\right)$ contains the arrow $\alpha_{i, i}$ and exactly one of $\alpha_{i, j}$ or $\beta_{i, j}$ if $i \neq j$. Therefore, there are two subcases.

2.1.1: For some $i$, for example $i=1, A_{1}\left(z_{1}, z_{2}\right)$ contains the arrow $\beta_{2,1}$. Then the union of the walks $A_{i}\left(i_{S}, z_{i}\right)$, for $i=1,2$ and $A_{1}\left(z_{1}, z_{2}\right)$ determines a subquiver of type $\tilde{\mathrm{A}}_{r}$ without relations, where $r$ is the sum of the lengths of those walks, which is illustrated by

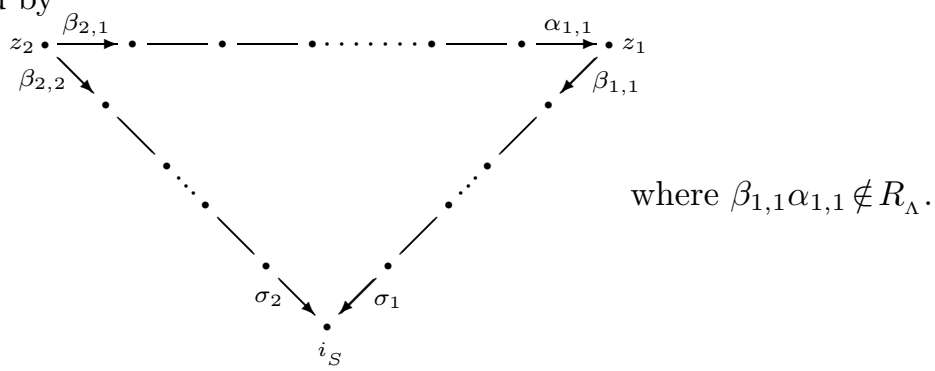

This contradicts the fact that $\Lambda$ is a representation-finite algebra.

2.1.2: For some $i$, for example $i=1, A_{1}\left(z_{1}, z_{2}\right)$ contains the arrow $\alpha_{2,1}$. Under this condition, $Q_{M}$ contains a subquiver which is the union of the walks $A_{i}\left(i_{S}, z_{i}\right)$, $i=1,2$, and $A_{1}\left(z_{1}, z_{2}\right)$, and it is illustrated by

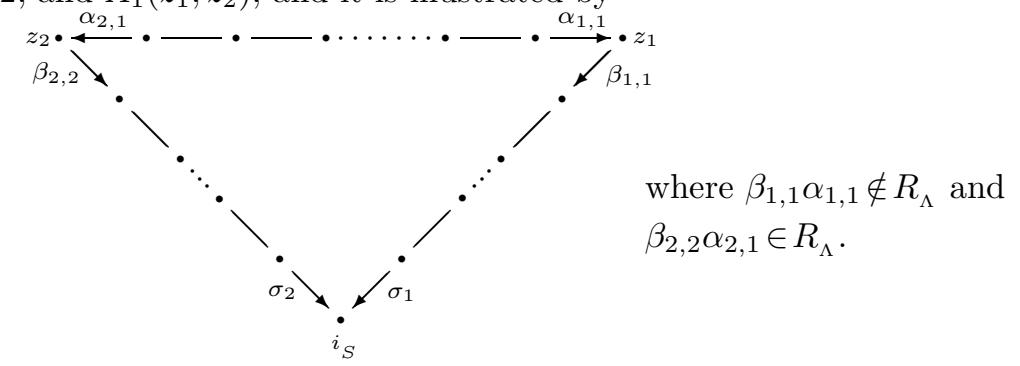

In this subcase, let $N^{\prime}=\left(N^{\prime}(x)_{x}, N^{\prime}(\alpha)_{\alpha}\right)$ and $N^{\prime \prime}=\left(N^{\prime \prime}(x)_{x}, N^{\prime \prime}(\alpha)_{\alpha}\right)$ be the families defined by

$$
N^{\prime}(x)= \begin{cases}N_{2}(x) & \text { if } x \in A_{2}\left(i_{S}, z_{2}\right), x \notin\left\{z_{2}, i_{S}\right\} \\ N_{1}(x) & \text { if } x \in A_{1}\left(i_{S}, z_{1}\right) \cup A_{1}\left(z_{1}, z_{2}\right) \text { and } x \neq i_{S}, \\ k v & \text { if } x=i_{S}, \\ (0) & \text { for the other vertices }\end{cases}
$$




$$
\begin{gathered}
N^{\prime}(\alpha)= \begin{cases}N_{2}(\alpha) & \text { if } \alpha \in A_{2}\left(i_{S}, z_{2}\right) \text { and } \alpha \neq \beta_{2,2}, \\
N_{1}(\alpha) & \text { if } \alpha \in A_{1}\left(i_{S}, z_{1}\right) \cup A_{1}\left(z_{1}, z_{2}\right), \\
0 & \text { for the other arrows, }\end{cases} \\
N^{\prime \prime}(x)= \begin{cases}N_{2}(x) & \text { if } x \in A_{2}\left(i_{S}, z_{2}\right) \text { and } x \neq i_{S}, \\
N_{1}(x) & \text { if } x \in A_{1}\left(i_{S}, z_{1}\right) \cup A_{1}\left(z_{1}, z_{2}\right) \text { and } x \notin\left\{z_{2}, i_{S}\right\}, \\
k v & \text { if } x=i_{S}, \\
(0) & \text { for the other vertices, }\end{cases} \\
N^{\prime \prime}(\alpha)= \begin{cases}N_{2}(\alpha) & \text { if } \alpha \in A_{2}\left(i_{S}, z_{2}\right), \\
N_{1}(\alpha) & \text { if } \alpha \in A_{1}\left(i_{S}, z_{1}\right) \cup A_{1}\left(z_{1}, z_{2}\right), \\
0 & \text { and } \alpha \neq \alpha_{2,1},\end{cases} \\
\end{gathered}
$$

It is easy to verify that $N^{\prime}$ and $N^{\prime \prime}$ are $k$-representations whose quivers are obtained from the quiver above by deleting the arrows $\beta_{2,2}$ and $\alpha_{2,1}$, respectively. Therefore, using the same arguments used in 2.1.2, we conclude that $N^{\prime}$ and $N^{\prime \prime}$ are indecomposable multiplicity-free modules having the same composition factors (since they have the same support), but they are not isomorphic (since $S_{z_{2}} \subset \operatorname{soc} N^{\prime}$ and $\left.S_{z_{2}} \not \subset \operatorname{soc} N^{\prime \prime}\right)$. Again we obtain a contradiction to the assumption on $\Lambda$-ind.

2.2: For some $i$, for example $i=1, A_{1}\left(i_{S}, z_{1}\right)$ contains the arrow $\alpha_{1,1}$. Then, $A_{1}\left(z_{1}, z_{2}\right)$ necessarily contains the arrow $\beta_{1,1}$. Relative to the other arrows to which $z_{1}$ and $z_{2}$ belong, we have the following subcases.

2.2.1: $A_{1}\left(z_{1}, z_{2}\right)$ contains the arrow $\beta_{2,1}$ (or $\alpha_{2,1}$ ) and $A_{2}\left(i_{S}, z_{2}\right)$ contains the arrow $\beta_{2,2}$ (respectively, $\alpha_{2,2}$ );

2.2.2: $A_{1}\left(z_{1}, z_{2}\right)$ contains $\beta_{2,1}$ and $A_{2}\left(i_{S}, z_{2}\right)$ contains $\alpha_{2,2}$; and

2.2.3: $A_{1}\left(z_{1}, z_{2}\right)$ contains $\alpha_{2,1}$ and $A_{2}\left(i_{S}, z_{2}\right)$ contains $\beta_{2,2}$. Since subcase 2.2 .1 is similar to subcase 2.1.1 and the others are similar to 2.1.2, we leave it to the reader to verify that they lead to a contradiction to the assumptions. Hence, we cannot suppose that $T$ does not have multiplicity one as a composition factor of $M$. So it is proved that each indecomposable module is multiplicity-free.

\section{REFERENCES}

1. I. Assem, D. Happel, Generalized tilted algebras of type $A_{n}$, Comm. in Algebra 9 (1981) 2101-2125; 10 (1982), 1475. MR 83a:16023a,b.

2. M. Auslander, Representation of algebras, notes of a course at Brandeis University, 1981.

3. M. Auslander, I. Reiten, Representation theory of Artin algebras III: Almost split sequences, Comm. in Algebra 3 (1975), 239-294. MR 52:504

4. M. Auslander, I. Reiten, Representation theory of Artin algebras V: Methods for computing almost split sequences and irreducible morphisms, Comm. in Algebra 5 (1977) 519-554. MR $\mathbf{5 5 : 1 2 7 6 3}$

5. M. Auslander, I. Reiten, Modules determined by their composition factors, Illinois J. Math. 29 (1985), 280-301. MR 86i: 16032

6. M. Auslander, I. Reiten, S. Smalø, Representation theory of Artin algebras, Cambridge University Press (1995). MR 96c: 16015

7. R. Bautista, On algebras of strongly unbounded representation type, Comm. Math. Helv. 60 (1985) 392-399. MR 87b:16029

8. C. Cibils, F. Larrión, L. Salmerón, Metodos diagramáticos en teoría de representaciones, in Monografías del Inst. de Matemáticas, 11 UNAM, Mexico (1982). MR 84d:16037

9. P. Gabriel, Auslander-Reiten sequences and representation-finite algebras, in Representation Theory I, Springer Lectures Notes in Math. 831 (1980) 1-71. MR 82i:16030

10. Ma. I. R. Martins, Fatores de Composição de Módulos Indecomponíveis, Tese de doutoramento na Universidade de São Paulo, Brasil (1994), 107 páginas. 
11. L. A. Nazarova, A. V. Rojter, Categorical matrix problems and the Brauer-Thrall conjecture, preprint, Inst. Math. Acad. Sci., Kiev 1973, German transl. Mitt. Math. Sem. Giessen 115 (1975). MR 54:360; MR 58:5790

12. Z. Pogorzály, A. Skowroński, On algebras whose indecomposable modules are multiplicity free, Proc. London Math. Soc. 47 (1983), 463-479. MR 85a:16034

13. C. M. Ringel, On algorithms for solving vector space problems. I: Report on the Brauer-Thrall conjectures, in: Representation Theory I, Lecture Notes in Mathematics 831 (1980), 104-136. MR 82j: 16055

14. A. Skowroński, Z. Waschbüsch, Representation-finite biserial algebras, J. Reine Ang. Math. 345 (1983), 172-181. MR 85e:16051

Departamento de Matemática-Imeusp, Universidade de São Paulo, CP 66281 - CeP 05315-970, São Paulo, Brazil

E-mail address: bel@ime.usp.br 